Journal of Fluid Mechanics

http://journals.cambridge.org/FLM

Journal of

Additional services for Journal of Fluid Mechanics:

Fluid Mechanics

Email alerts: $\underline{\text { Click here }}$

Subscriptions: $\underline{\text { Click here }}$

Commercial reprints: $\underline{\text { Click here }}$

Terms of use : $\underline{\text { Click here }}$

\title{
Dispersive effects in Rossby-wave hydraulics
}

E. R. JOHNSON and S. R. CLARKE

Journal of Fluid Mechanics / Volume 401 / December 1999, pp 27 - 54

DOI: 10.1017/S0022112099006849, Published online: 08 September 2000

Link to this article: http://journals.cambridge.org/abstract_S0022112099006849

How to cite this article:

E. R. JOHNSON and S. R. CLARKE (1999). Dispersive effects in Rossby-wave hydraulics. Journal of Fluid Mechanics, 401, pp 27-54 doi:10.1017/S0022112099006849

Request Permissions : $\underline{\text { Click here }}$ 


\title{
Dispersive effects in Rossby-wave hydraulics
}

\author{
By E. R. JOHNSON AND S. R. CLARKE $\dagger$ \\ Department of Mathematics, University College London, Gower Street, \\ London, WC1E 6BT, UK
}

(Received 22 May 1998 and in revised form 3 June 1999)

This paper considers the role of long finite-amplitude Rossby waves in determining the evolution of flow along a rapidly rotating channel with an uneven floor. The Rossby waves travel on a potential vorticity interface in a channel with a crosschannel step change in depth, where step position varies slowly along the channel. A nonlinear wave equation is derived describing the evolution of the potential vorticity interface. To leading order this is the hydraulic equation derived by Haynes, Johnson \& Hurst (1993). Dispersion appears at the next order. Various solution regimes are identified. As well as slowly varying hydraulic solutions, two further types of steady solutions appear: approach-controlled flows and twin supercritical leaps. Both these solutions are characterized by leaps between supercritical branches of the hydraulic function. It is shown how the position and size of these 'supercritical leaps' can be determined within the context of hydraulic theory. To fully resolve the internal structure of these leaps dispersive effects must be included and leaps are shown to correspond to kink soliton solutions of the steady unforced problem. It is also shown that increasing dispersion (decreasing topographic length scale) causes the loss of the subcritical solution branch in some subcritical flows. The only candidate for a steady solution in these regimes is then an approach-controlled flow. Integrations of initial value problems show that in general flows evolve towards the dispersive form of the solution predicted by hydraulic theory, at least near the topographic perturbation. However, in those subcritical flows where sufficiently large dispersion causes the subcritical branch to disappear, unsteady integrations evolve to approach-controlled flows even when the dispersion is sufficiently small that the subcritical branch still exists.

\section{Introduction}

The nonlinear flow response to slowly varying perturbations is often largely determined by the local strength of the perturbation to the flow. The perturbation can be contractions in the width of a waveguide or changes in the fluid depth. One of the most successful examples of slowly varying theory is the hydraulic theory of open channel flow. Baines (1995) gives a review of this theory and also its extension to interfacial and internal waves in two-layer and continuously stratified flows.

Regions of flow are described as supercritical or subcritical depending on whether the local flow speed is greater or less than some intrinsic wave speed. In a rotating fluid hydraulic behaviour can occur for 'fast' modes like Kelvin waves and for 'slow' modes like Rossby waves and coastally trapped waves. Studies of the hydraulics of

$\dagger$ Present address: Department of Mathematics and Statistics, Monash University, Clayton 3168, Australia. 
Kelvin waves are reviewed in Pratt \& Lundberg (1991). The hydraulics of slow modes over coastal topography and in straits has been considered by, among others, Hughes (1985a, b, 1986a, b, 1987, 1989), Pratt \& Armi (1987), Pratt \& Lunderberg (1991), Stern (1993) and Woods (1993).

One of the simplest configurations that supports Rossby waves is a rotating channel with a cross-channel step change in depth. Topographic Rossby waves can be generated in this system when a uniform flow along the channel encounters a perturbation to the position of the step. This geometry was considered by Haynes, Johnson \& Hurst (1993, referred to hereinafter as HJH), who used hydraulic theory, both steady and unsteady, and contour-dynamical (CD) simulations to study hydraulic control and upstream influence. They showed that, in addition to fully subcritical and supercritical flows, the flow could behave like hydraulically controlled open-channel flow, being subcritical upstream of the maximum topographic perturbation and supercritical downstream. HJH also described an unusual controlled flow where the long-wave speed vanishes at the leading edge of the topographic perturbation. The flow is supercritical downstream of the leading edge and jumps from one supercritical branch of the hydraulic solution to another downstream of the maximum topographic perturbation. It was not clear what determined the form and position of this supercritical jump. For moderately long obstacles, where the length was longer than the channel width, the agreement between the CD simulations of $\mathrm{HJH}$ and the steady hydraulic solutions was generally excellent near the topographic perturbations. Away from the perturbations free waves with significant amplitudes, absent from hydraulic theories, appeared. The discussion in HJH was limited to the case where the position of the step far upstream and downstream, $Y_{0}$, was midway between the sides of the channel. A weakly nonlinear equation was derived by $\mathrm{HJH}$ to describe the near-critical forcing for general $Y_{0}$. When $Y_{0}$ is mid-channel the quadratic nonlinearity in this equation vanishes and only a odd nonlinear term remains. As $Y_{0}$ moves towards either side of the channel quadratic nonlinearity dominates. Studies of the weakly nonlinear forcing of a two-layer fluid by flow over topography show that the behaviour is very different when the initial position of the thermocline is mid-depth (Melville \& Helfrich 1987; Marchant \& Smyth 1990), and so asymmetrically placed steps could lead to markedly different flows from the symmetrically placed steps of HJH.

It is the purpose of this paper to investigate in greater analytical and numerical detail the behaviour of the flows in HJH. In the course of this investigation it will be shown that the choice there of a symmetrically placed step is special and new behaviour is found for asymmetrically placed steps. Steady and unsteady transient and forced wavetrains are described and a complete specification of the structure and position of the supercritical jump of $\mathrm{HJH}$ given. It is shown that the transition corresponds directly to the 'supercritical leap' (Baines 1995) identified in stratified, two-layer non-rotating flow by Lawrence (1993) and Baines (1984). Progress is made possible by introducing the leading-order effects of dispersion into the hydraulic equations and thus retaining dispersive long-wave dynamics in a finite-amplitude evolution equation. Similar dispersive effects, based on the study of finite-amplitude waves on coastal currents by Grimshaw \& Yi (1990), are introduced in Clarke \& Johnson $(1997 a, b)$ to model the CD simulations of a coastal current in Stern (1991). An important advantage of the resulting evolution equation is that numerical integration is an order of magnitude faster than $\mathrm{CD}$ integrations, allowing a more comprehensive exploration of the parameter space. In Clarke \& Johnson (1999, referred to hereinafter as $\mathrm{CJ}$ ), the introduction of leading-order dispersion allowed discussion of the steady free-wave solutions of the unforced system of HJH. These 


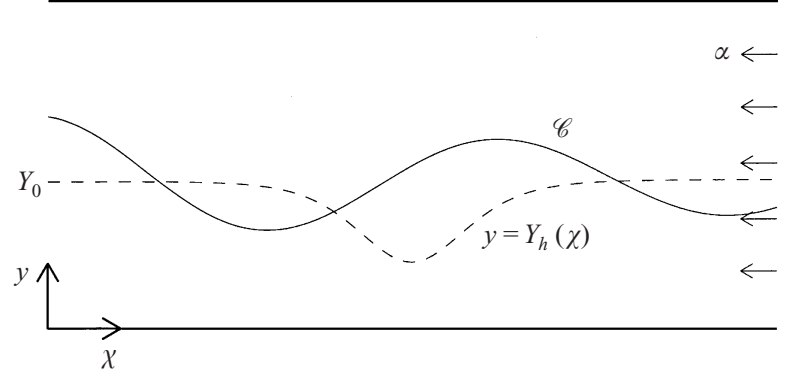

Figure 1. The non-dimensionalized geometry. The channel has width 1 and the step edge (shown dashed here and in subsequent figures) is at $y=Y_{h}(\chi)$ with the shallower, shelf, region in $y<Y_{h}$. Here $\chi=\mu^{1 / 2} x$ is the along-channel distance scaled on the channel width and $\mu^{1 / 2} \ll 1$. Far upstream and downstream $Y_{h} \rightarrow Y_{0}$. The maximum deviation of the shelf edge from $Y_{0}$ is given by $-\epsilon$ and is not required to be small. The oncoming flow has speed $\alpha$ from right to left. The material interface $\mathscr{C}$ (shown as a solid line here and subsequently) separates fluid with potential vorticity 1 from fluid with potential vorticity 0 . If this is a single-valued function of $\chi$ it can be written as $y=Y$, and then in $y>Y(\chi)$ fluid has potential vorticity 0 and in $y<Y(\chi)$ fluid has potential vorticity 1 .

solutions correspond to the finite-amplitude waves observed by $\mathrm{HJH}$ away from the topographic perturbation and also describe aspects of the behaviour near the topography.

In terms of the parameter $\mu^{1 / 2}$, giving the ratio of the channel width to the scale of along-channel variations in shelf-width, hydraulic theory corresponds to the limit $\mu \rightarrow 0$, and topographic variations appear only parametrically. Section 2 considers the first effects of finite-length topography for $0<\mu \ll 1$, showing that these introduce dispersion into the wave dynamics, and deriving the unsteady, forced dispersive wave equation. Section 3 discusses flow behaviour in the hydraulic limit $(\mu \rightarrow 0)$ of arbitrarily long obstacles, obtaining the size and position of supercritical leaps and an apparently novel flow type with a twin supercritical leap. Section 4 describes steady dispersive flows $(0<\mu \ll 1)$, noting the finite-amplitude Rossby-wave wakes in subcritical flow, the internal structure of the supercritical leaps in supercritical flow, and the loss of the subcritical solution branch with increasing $\mu$ in some regions of parameter space. Section 5 describes the unsteady dispersive evolution of flows from given initial conditions for three representative choices of $Y_{0}$. The results are discussed briefly in $\S 6$.

\section{The dispersive evolution equation}

Consider inviscid flow in a rotating channel of constant width $L$ and mean depth $H_{0}$, with a rigid upper surface and variable bottom topography as shown in figure 1. Let the rotation be sufficiently fast and the bottom topography sufficiently low $\dagger$ that the flow is two-dimensional with governing equation the non-dimensional (quasigeostrophic) conservation of potential vorticity (PV):

$$
\frac{\partial q}{\partial t}+\frac{\partial(\Psi, q)}{\partial(x, y)}=0
$$

$\dagger$ The extension to step heights of order the fluid depth is straightforward and follows Bidlot \& Stern (1994). 
where $q$ is the PV scaled on $Q,(x, y)$ are Cartesian coordinates scaled on $L, t$ is time scaled on $Q^{-1}$, and $\Psi$ is a streamfunction, scaled on $Q L^{2}$. The PV is thus

$$
Q q=Q \nabla^{2} \Psi+\frac{f_{0} h}{H_{0}}
$$

where $f_{0}$ is the Coriolis parameter and $h$ the height of the bottom topography.

Let the channel walls be at $y=0,1$ and consider a flat shelf with a downward $\dagger$ step change $h_{0}$ in depth at the shelf edge $y=Y_{h}(\chi)$. Here $\chi$ gives the scale of along-channel variations in the shelf and is currently unrestricted. The relationship between $\chi$ and $x$ gives the small parameter, $\mu$, for the subsequent analysis. In particular let $\chi=\mu^{1 / 2} x$, and $\tau=\mu^{1 / 2} t$. The step shelf can thus be written $h=h_{0} H\left(1-Y_{h}\right)$, where $H(\cdot)$ is the Heaviside step function. For simplicity the change in the shelf width is taken here to have a single, isolated extremum so $Y_{h}(\chi) \rightarrow Y_{0}$ (say) as $\chi \rightarrow \pm \infty$.

Let the fluid be at rest at $t=0$. At $t=0^{+}$a constant, down-channel (in the negative$x$ direction), non-dimensional, volume flux $\alpha$ is imposed and then maintained for all $t>0$. The initial PV distribution can thus be written as $q=H\left(1-Y_{h}\right)$, where the previously undetermined PV scale $Q$ has been taken equal to the PV generated by topographic vortex stretching, $f_{0} h_{0} / H_{0}$. The imposed flow causes the material interface $\mathscr{C}$ separating the two regions of piecewise-constant potential vorticity to be advected from its initial position $Y_{h}(\chi)$. Fluid moving off the shelf gains positive relative vorticity while fluid moving onto the shelf gains negative relative vorticity. Provided $\mathscr{C}$ remains a single-valued function of $x$ its position can be written as $y=Y(\chi, \tau)$. Writing the total streamfunction as a mean flow component and a perturbation streamfunction $\psi$,

$$
\Psi(\chi, y, \tau)=\alpha y+\psi(\chi, y, \tau)
$$

allows the evolution of the flow to be described in terms of

$$
\psi_{y y}+\mu \psi_{\chi \chi}=H\left(y-Y_{h}\right)-H(y-Y),
$$

where

$$
\begin{aligned}
\psi & =0 \quad \text { on } y=0,1, \\
Y_{\tau} & =\frac{\partial}{\partial \chi}\{\alpha Y(\chi, \tau)+\psi(\chi, Y(\chi, \tau), \tau)\}, \\
Y(\chi, 0) & =Y_{h}(\chi) .
\end{aligned}
$$

So far no restrictions have been placed on the size of the topographic perturbation. Analytical progress can be made by limiting attention to infinitesimal perturbations in the shelf width; however, the resulting linear wave problems miss much of the remarkable nonlinear behaviour of the flow. Here no restriction will be placed on the amplitude of the deviation of the shelf edge from its upstream value, rather the analysis will be simplified by considering shelves whose width varies only slowly along the channel, i.e. by taking $\mu \ll 1$. The CD integrations in HJH then correspond to arbitrary $\mu$ and the hydraulic theory to the limit $\mu \rightarrow 0$. Analytical results will be extended to small but finite $\mu$, introducing the leading-order effects of dispersion to the theory of HJH. Seeking a perturbation solution of the form

$$
\psi=\psi^{(0)}+\mu \psi^{(1)}+\cdots
$$

$\dagger$ Results for upward steps follow by noting that the transformation $x \rightarrow-x, y \rightarrow 1-y$ maps a downward step to an upward one. 
gives the leading-order equation

$$
\psi_{y y}^{(0)}=H\left(y-Y_{h}\right)-H(y-Y) .
$$

Each term in the expansion for $\psi$ satisfies the homogeneous boundary condition $(2.4 b)$ and so

$$
\begin{aligned}
\psi^{(0)}=\frac{1}{2} H\left(y-Y_{h}\right)\left(y-Y_{h}\right)^{2}-\frac{1}{2} H(y-Y)(y-Y)^{2}+\frac{1}{2}\left((1-Y)^{2}-\left(1-Y_{h}\right)^{2}\right) y, \\
\psi^{(1)}=-\frac{1}{12} \frac{\partial^{2}}{\partial \chi^{2}}\left\{\frac{1}{2} H\left(y-Y_{h}\right)\left(y-Y_{h}\right)^{4}-\frac{1}{2} H(y-Y)(y-Y)^{4}\right. \\
\left.+\left((1-Y)^{2}-\left(1-Y_{h}\right)^{2}\right)\left(y^{3}-y\right)+\frac{1}{2}\left((1-Y)^{4}-\left(1-Y_{h}\right)^{4}\right) y\right\} .
\end{aligned}
$$

The dynamic interface condition $(2.4 c)$ gives, with $(2.4 d)$, the initial value problem

$$
\begin{aligned}
& Y_{\tau}= \frac{\partial}{\partial \chi}\left\{\alpha Y+\frac{1}{2} H\left(Y-Y_{h}\right)\left(Y-Y_{h}\right)^{2}+\frac{1}{2}\left((1-Y)^{2}-\left(1-Y_{h}\right)^{2}\right) Y\right. \\
&+\frac{\mu}{6}\left[H\left(Y-Y_{h}\right)\left(Y-Y_{h}\right)^{2}\left(Y_{h \chi \chi}\left(Y-Y_{h}\right)-3 Y_{h \chi}^{2}\right)\right. \\
&-Y\left[Y_{h \chi \chi}\left(1-Y_{h}\right)\left(Y^{2}-1+\left(1-Y_{h}\right)^{2}\right)-Y_{h \chi}^{2}\left(Y^{2}-1+3\left(1-Y_{h}\right)^{2}\right)\right] \\
&\left.\left.-2 Y(1-Y) \frac{\partial}{\partial \chi}\left\{Y(1-Y) Y_{\chi}\right\}\right]\right\}+O\left(\mu^{2}\right), \\
& Y(\chi, 0)=Y_{h}(\chi) .
\end{aligned}
$$

At leading order this is the hyperbolic system studied in HJH. This paper considers solutions of (2.9) for topography of the form

$$
Y_{h}=Y_{0}-\epsilon f(\chi),
$$

where $f$ has maximum unity and minimum zero. Numerical simulations use the particular choice

$$
Y_{h}=Y_{0}-\epsilon \operatorname{sech}^{2} \chi
$$

As (2.4), and consequently (2.9), is invariant under the transformation $\left(\psi, y, Y, Y_{h}\right) \rightarrow$ $1-\left(\psi, y, Y, Y_{h}\right)$, it is sufficient to consider only narrowings of the shelf, i.e. $0 \leqslant \epsilon<Y_{0}$.

\section{The hydraulic limit}

The leading-order (in $\mu$ ) approximation to (2.9) is the unsteady hydraulic equation

$$
\frac{\partial Y}{\partial \tau}-\frac{\partial \Psi_{h}}{\partial Y} \frac{\partial Y}{\partial \chi}=\frac{\partial \Psi_{h}}{\partial Y_{h}} \frac{\partial Y_{h}}{\partial \chi},
$$

where

$$
\Psi_{h}\left(Y, Y_{h}\right)=\alpha Y+\frac{1}{2} Y\left[(1-Y)^{2}-\left(1-Y_{h}\right)^{2}\right]+\frac{1}{2}\left(Y-Y_{h}\right)^{2} H\left(Y-Y_{h}\right) .
$$

Equation (3.1) is a forced first-order wave equation, giving the long-wave speed (rightwards positive) for small disturbances to the interface $\mathscr{C}$ as

$$
c(\chi)=-\frac{\partial \Psi_{h}\left(Y, Y_{h}\right)}{\partial Y} .
$$


The flow is supercritical in regions where $c<0$ with information carried downstream to the left along the interface and subcritical where $c>0$ with information carried upstream. In general the wave speed depends on both the position of the interface, $Y$, and the position of the shelf edge, $Y_{h}$. However, for infinitesimal waves, where both $Y$ and $Y_{h}$ differ negligibly from $Y_{0}$, the upstream position of the shelf edge, the speed $c$ reduces to the difference between the flow speed $\alpha$ and the infinitesimal long-wave speed $Y_{0}\left(1-Y_{0}\right)$. A Froude number for the flow can thus be introduced as

$$
F r=\alpha /\left[Y_{0}\left(1-Y_{0}\right)\right],
$$

with $\mathrm{Fr}>1$ corresponding to $c>0$ and supercritical small-amplitude flow and $\mathrm{Fr}<1$ corresponding to $c<0$ and subcritical small-amplitude flow.

The function $\Psi_{h}\left(Y, Y_{h}\right)$ is precisely the hydraulic function $G\left(Y, Y_{h}\right)$ discussed in HJH and CJ. Steady hydraulic solutions satisfy, from (3.1),

$$
\Psi_{h}\left(Y(\chi), Y_{h}\right)=\Psi_{0},
$$

for some constant $\Psi_{0}$, the value of the streamfunction on the steady interface $\mathscr{C}$. HJH show how these solutions for a given flow speed $\alpha$ can be represented as contours of the hydraulic function $\Psi_{h}$ in $\left(Y, Y_{h}\right)$-space, plots referred to here as hydraulic diagrams. Figure 2 shows the hydraulic diagrams and interface displacement for the four types of flow identified in $\mathrm{HJH} \dagger$.

Since $\partial \Psi_{h} / \partial Y_{h}$ is positive, supercritical regions correspond to sections of trajectories on the hydraulic diagram with negative slope and subcritical flows to sections with positive slope. The slope becomes vertical at critical points where $c$ vanishes. Completely subcritical and completely supercritical flows are localized, upstream-downstream symmetric flows (for symmetric topography), corresponding to monotonic trajectories. In both these flows $Y \rightarrow Y_{0}$ as $\chi \rightarrow \infty$ so the trajectory passes through $\left(Y_{0}, Y_{0}\right)$ and $\Psi_{0}=\alpha Y_{0}$. For constriction-controlled (CC) solutions the long-wave speed is zero at the maximum topographic perturbation (where $Y_{h}=Y_{\epsilon}$ ), positive upstream and negative downstream. The flow is subcritical upstream of the control, critical at the control and supercritical downstream. The interface streamfunction value $\Psi_{0}$ is thus determined by requiring that $c$ vanishes, i.e. $\left(\Psi_{h}\right)_{Y}=0$, when $Y_{h}=Y_{\epsilon}$.

\subsection{Approach-controlled flows and supercritical leaps}

For approach-controlled (AC) flows the long-wave speed vanishes at the upstream, leading, edge of the obstacle. The trajectory must pass through $Y_{h}=Y_{\epsilon}$ but in this parameter regime no subcritical branch attains this value of $Y_{h}$. The flow is thus supercritical downstream of the leading edge. The value of $\Psi_{0}$ on AC trajectories is determined by requiring that $c$ vanishes, i.e. $\left(\Psi_{h}\right)_{Y}=0$, at the leading edge. $\mathrm{HJH}$ note that although for symmetric topography a completely symmetrical supercritical AC solution exists with

$$
\lim _{x \rightarrow-\infty} Y=\lim _{x \rightarrow \infty} Y \neq Y_{0},
$$

this is not observed in contour dynamical integrations of the initial value problem. Downstream of the maximum topographic perturbation the flow falls to a different supercritical branch. In discussions of stratified two-dimensional two-layer nonrotating flow over ridges Lawrence (1993) and Baines (1984) described a flow type (termed approach-controlled flow by Lawrence) that is closely analogous to this $\mathrm{AC}$

$\dagger$ The hydraulic diagrams here are reflected about the line $Y=Y_{h}$ compared to those in $\mathrm{HJH}$ so as to be more closely comparable to those usually presented for stratified flows. 

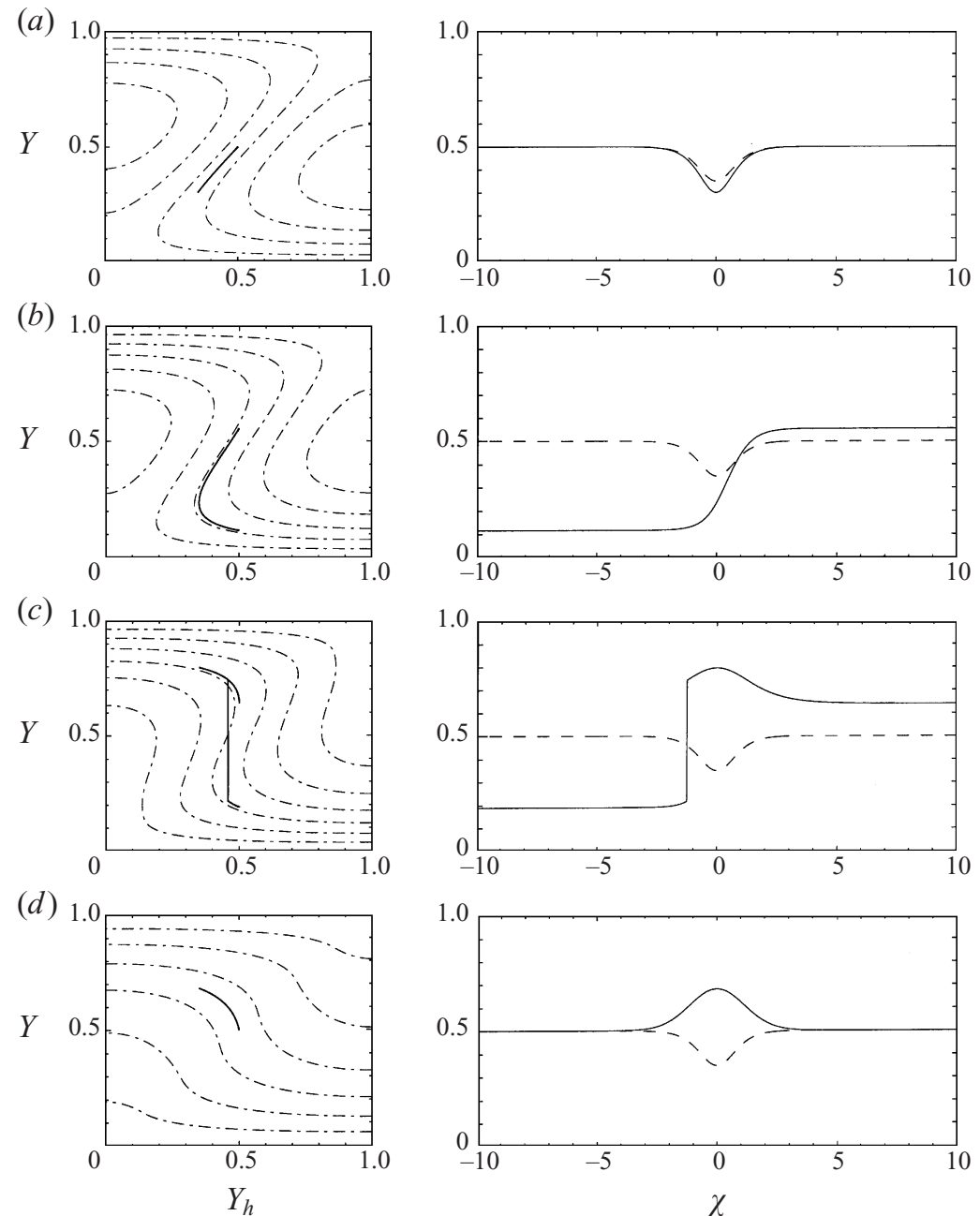

FIGURE 2. Examples of the four types of hydraulic solutions of (3.5) considered by HJH for $Y_{0}=0.5$ : (a) subcritical $(\epsilon=0.15, \alpha=0.05$, so $F r=0.2)$, $(b)$ constriction-controlled $(\epsilon=0.15, \alpha=0.1$, so $F r=0.4),(c)$ approach-controlled $(\epsilon=\alpha=0.15$, so $F r=0.6)$ and $(d)$ supercritical $(\epsilon=0.15, \alpha=0.3$, so $F r=1.2$ ). The left-hand part of each example gives the hydraulic diagram, with contours of $\Psi_{h}$ shown here, and in subsequent hydraulic diagrams, as dash-dotted lines. These give possible steady hydraulic flows. The chosen solution (discussed in the text) is shown (here and subsequently) by the bold solid line. The right-hand part of each example shows the position of the interface $\mathscr{C}$ (solid line) in the channel geometry for the shelf edge (dashed line) (2.11).

flow with its transition between supercritical branches and so, following the stratified flow convention (Baines 1995), these transitions are described as supercritical leaps (SLs) here. The reason for the SL in AC flows in $\mathrm{HJH}$, its position, and its structure are not considered there. However, straightforward considerations allow the SL to be completely fixed. Let $Y_{s}(\chi)$ be a steady solution of (3.1) so $Y_{s}(\chi)$ satisfies (3.5). Now consider the evolution of a small perturbation $\delta(\chi, \tau)$ to $Y_{s}(\chi)$. Equation (3.1) gives

$$
\delta_{\tau}=\left[\Psi_{h}\left(Y_{s}+\delta, Y_{h}\right)\right]_{\chi}=\left[\Psi_{0}-c(\chi) \delta\right]_{\chi}+O\left(\delta^{2}\right),
$$


with $c$ defined by (3.3) and evaluated on $Y=Y_{s}$. To leading order this gives, on multiplying by $c$,

$$
(c \delta)_{\tau}=-c(c \delta)_{\chi} .
$$

On characteristics $\mathrm{d} \chi / \mathrm{d} \tau=c(\chi)$, the quantity $c \delta$ (or any function of $c \delta$ ) is conserved. It is related to the momentum of the perturbation and (3.8) is the equation for the conservation of disturbance momentum, with disturbance momentum carried downstream in supercritical flow and upstream in subcritical flow, as expected. The general solution of (3.8) can be written, for an arbitrary differentiable function $F$, as

$$
\delta=\frac{1}{c} F\left(\tau-\int \frac{\mathrm{d} \chi}{c(\chi)}\right) .
$$

Momentum conservation (and the solution (3.9)) requires that the amplitude of a perturbation increases as its propagation speed decreases and becomes arbitrarily large approaching a point where the speed vanishes. In a AC flow without an SL a small perturbation to the material interface introduced just downstream of the leading edge of the topography would be swept downstream, first shrinking in amplitude as $-c$ increased to its maximum at the topography maximum and then growing arbitrarily large as $-c$ decreased to zero at the trailing edge of the topography. The interface position would be convectively unstable. The AC flow without an SL would not be observed as the final state of an initial value problem. In general an SL is required in a flow wherever the perturbation speed decreases to zero in the direction of propagation.

Lawrence (1993) noted that a stratified SL did not require a shock or internal hydraulic jump. Rather, its dynamics are non-hydrostatic. He also suggested that the exact position of the internal hydraulic control is weakly dependent on frictional forces. The dispersive terms of (2.9) are equivalent in the present Rossby-wave problem to non-hydrostatic terms in the stratified flow and are shown in $\S 4$ to give the internal structure of the transition. However the SL, which is smooth over alongchannel scales of order the channel width, is a step change in the interface position on along-channel scales of order of changes in the topography and its position and size can be determined from the hydraulic solution alone. In terms of the total streamfunction $\Psi$ the requirement that the flow is steady becomes a requirement that $\Psi$ is constant along the interface, i.e.

$$
\Psi(x, Y(x))=\Psi_{0},
$$

for some constant $\Psi_{0}$. Differentiating (3.10) gives

$$
\frac{\mathrm{d}}{\mathrm{d} x}\{\Psi(x, Y(x))\}=0 .
$$

Multiplying (3.11) by $Y(x)$ and integrating with respect to $x$ from $x_{-}$to $x_{+}$gives

$$
\begin{aligned}
0=\int_{x_{-}}^{x_{+}} Y(x) \frac{\mathrm{d}}{\mathrm{d} x}\{\Psi(x, Y(x))\} \mathrm{d} x & =-\int_{x_{-}}^{x_{+}} \Psi Y_{x} \mathrm{~d} x+\Psi_{0}\left(Y_{+}-Y_{-}\right) \\
& =-\int_{Y_{-}}^{Y_{+}} \Psi\left(Y, Y_{h}(x)\right) \mathrm{d} Y+\Psi_{0}\left(Y_{+}-Y_{-}\right)
\end{aligned}
$$

where $Y_{-}=Y\left(x_{-}\right)$and $Y_{+}=Y\left(x_{+}\right)$and to move to the final expression of (3.12) it is necessary that $Y(x)$ is a monotonic function of $x$ in the region $x_{-}<x<x_{+}$.

So far no assumption has been made about the abruptness of the transition. Now 


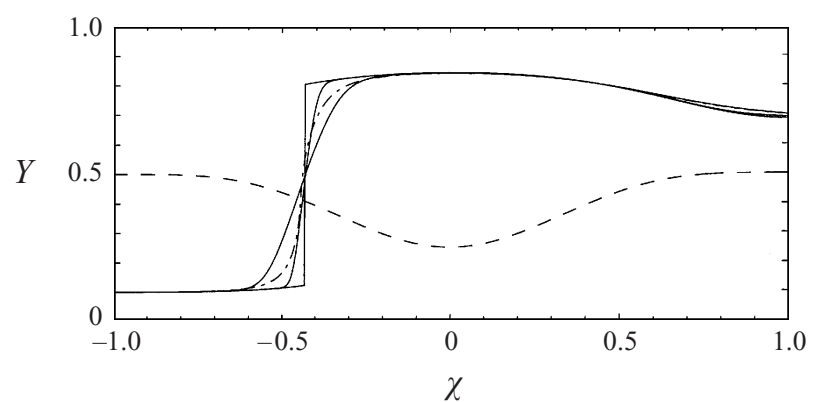

FiguRE 3. A comparison of the approach-controlled CD solution of HJH11(c), shown as a dash-dotted line, with steady solutions of the finite-amplitude dispersive equation (2.9) and the limiting supercritical leap solution satisfying (3.13). Here $Y_{0}=0.5, \epsilon=0.25, \alpha=(3 \pi)^{-1}$ (so $F r \approx 0.42$ ). The topographic perturbation has finite support, with $f=\cos ^{4}\left(\frac{1}{2} \pi \chi\right)$ for $|\chi|<1$ and vanishing otherwise, and the shelf edge is shown dashed. The three solid curves are for the values $\mu \approx 0.0386$, 0.0039 and zero. The geometry of HJH11(c) corresponds to the largest dispersion and it can be seen that except in the immediate neighbourhood of the SL the relevant finite-amplitude solution reproduces the $\mathrm{CD}$ integration accurately, particularly near $\chi=1$ where $f^{(\mathrm{iv})}$ is discontinuous.

assume that the transition takes place over a distance small compared to the scale of changes in the shelf width. This will always be true for sufficiently small $\mu$. Then to order $\mu, Y_{h}(x)$ can be taken to be constant, $Y_{h}^{T}$, and $\Psi$ can be replaced by the hydraulic form $\Psi_{h}$, giving the required jump conditions,

$$
\begin{aligned}
\Psi_{h}\left(Y_{-}, Y_{h}^{T}\right) & =\Psi_{0}, \\
\Psi_{h}\left(Y_{+}, Y_{h}^{T}\right) & =\Psi_{0}, \\
\int_{Y_{-}}^{Y_{+}} \Psi_{h}\left(Y, Y_{h}^{T}\right) \mathrm{d} Y & =\Psi_{0}\left(Y_{+}-Y_{-}\right) .
\end{aligned}
$$

The multiplicative function chosen in moving from (3.11) to (3.12) is arbitrary in the sense that any function of $Y$ alone could be chosen. The particular choice is motivated in $\S 4$ by considering the form of the solution within the jump. The three unknowns in (3.13) are $Y_{-}$and $Y_{+}$, the heights either side of the SL, and $Y_{h}^{T}$, the topographic displacement at the SL (which fixes the position through (2.10)). The three polynomial matching relations (3.13) determine them uniquely. In the present inviscid flow the position of the SL is fixed and so in slightly viscous flow the position could be expected to vary only weakly with frictional forces as noted by Lawrence (1993) in stratified flow. Figure 3 gives a comparison between the AC flow in a long-time $\mathrm{CD}$ solution of the full equations taken from figure 11(c) of $\mathrm{HJH}$ (denoted HJH11(c) herein) and results from the finite-amplitude equation (2.9). These solutions are obtained by approximating the steady form of (2.9) by finite differences and solving the resulting nonlinear algebraic equations by Newton's method as noted in the Appendix. The CD integration is shown dash-dotted and three solutions of (2.9) are shown. The solution with the vertical jump is the limit $\mu \rightarrow 0$ of the dispersive solutions and the position of the jump agrees precisely with the solution of (3.13).

\subsection{Twin supercritical leaps}

For $Y_{0}=\frac{1}{2}$ the four flow types noted in $\mathrm{HJH}$ completely describe the hydraulic behaviour of the system. However, in the range (whose bounds are derived below) where $0.0938<Y_{0}<\frac{1}{3}$, a regime exists where none of the four flow types identified in 

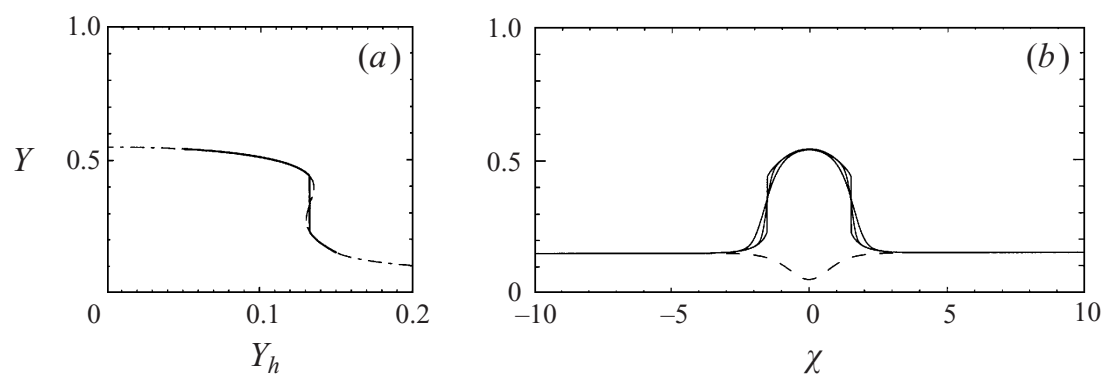

FiguRE 4. A twin supercritical leap (TSL) solution of the hydraulic equation (3.5) for $Y_{0}=0.15$, $\epsilon=0.1$ and $\alpha=0.17$ (so $F r \approx 1.33$ ). (a) The hydraulic diagram with the TSL solution shown bold and the $\Psi_{h}$ contour from which it is constructed shown as a dash-dotted line. (b) The TSL solution in the channel geometry for the shelf-edge (dashed) (2.11) and steady solutions of the long-wave dispersive equation (2.9) with $\mu=0.01$ and 0.1 . The hydraulic TSL solution corresponds to the limit $\mu \rightarrow 0$. As dispersion $(\mu)$ decreases the slopes of the SLs increase.

$\mathrm{HJH}$ are possible and a new type occurs. To see this consider the hydraulic contour shown in figure $4(a)$. Subcritical flow can be eliminated as the flow is well within the upstream-supercritical region and contour slopes are negative at $\left(Y_{0}, Y_{0}\right)$. CC flow can be eliminated as the critical contour (with $\left(\Psi_{h}\right)_{Y}=0$ at $Y_{h}=Y_{\epsilon}$ and $\left.\Psi_{h}<\alpha Y_{0}\right)$ cuts $Y_{h}=Y_{0}$ only once. AC flow can be eliminated as the contour with $\left(\Psi_{h}\right)_{Y}=0$ at $Y_{h}=Y_{0}$ (and $\left.\Psi_{h}>\alpha Y_{0}\right)$ intersects $Y_{h}=Y_{0}$ again in $Y>Y_{0}$. Thus the downstream level would be greater than $Y_{h}$, i.e. the flow would not cross the step. Finally, purely supercritical flow can be eliminated as no monotonic trajectory joins $Y_{h}=Y_{0}$ to $Y_{h}=Y_{\epsilon}$. The remaining possibility is the trajectory shown in bold, where the flow passes through an SL between two supercritical branches of the contour. The flow is symmetric with two SLs, one upstream and one downstream of the maximum topographic perturbation. This type of flow is termed a twin supercritical leap (TSL) here. The flow can be described in the physical terms used for AC flows. The flow lies within the general region of upstream-supercritical flows with information carried downstream from $\chi \gg 1$. The hydraulic contour is thus undisturbed upstream and passes through $\left(Y_{0}, Y_{0}\right)$. The speed $-c$ of a small disturbance moving along this contour in the flow direction decreases as $Y_{h}$ decreases and would vanish before $Y_{h}$ reached $Y_{\epsilon}$. As noted above this would lead to small disturbances growing arbitrarily. The flow thus jumps to the alternative, larger- $Y$, supercritical branch and continues to $Y_{h}=Y_{\epsilon}$. As $Y_{h}$ increases after the topographic extremum the solution retraces its path along the upper- $Y$ supercritical branch. Before $Y_{h}$ can return to $Y_{0}$ a second point where $c$ vanishes is approached and to avoid the singularity the solution jumps back to the original, lower- $Y$, supercritical branch. Figure $4(b)$ shows the TSL solution of figure $4(a)$ for the topography (2.11). Also shown are two steady solutions of the finite-amplitude equation (2.9) with $\mu>0$ obtained by the Newton method of the Appendix. As in figure 3, dispersion smooths the supercritical transition but the hydraulic solution with the jump conditions (4.7) accurately predicts the position of the transition in the finite- $\mu$ solutions and the behaviour outside the immediate neighbourhood of the transition.

\subsection{Hydraulic regime diagrams}

Figure 5 gives regime diagrams for seven values of $Y_{0}$ showing the regions of the $(\alpha, \epsilon)$ parameter space associated with the different types of hydraulic solution. The axes also show the values of the alternative pair $(F r, B)$ where $B=\epsilon / Y_{0}$ gives the 

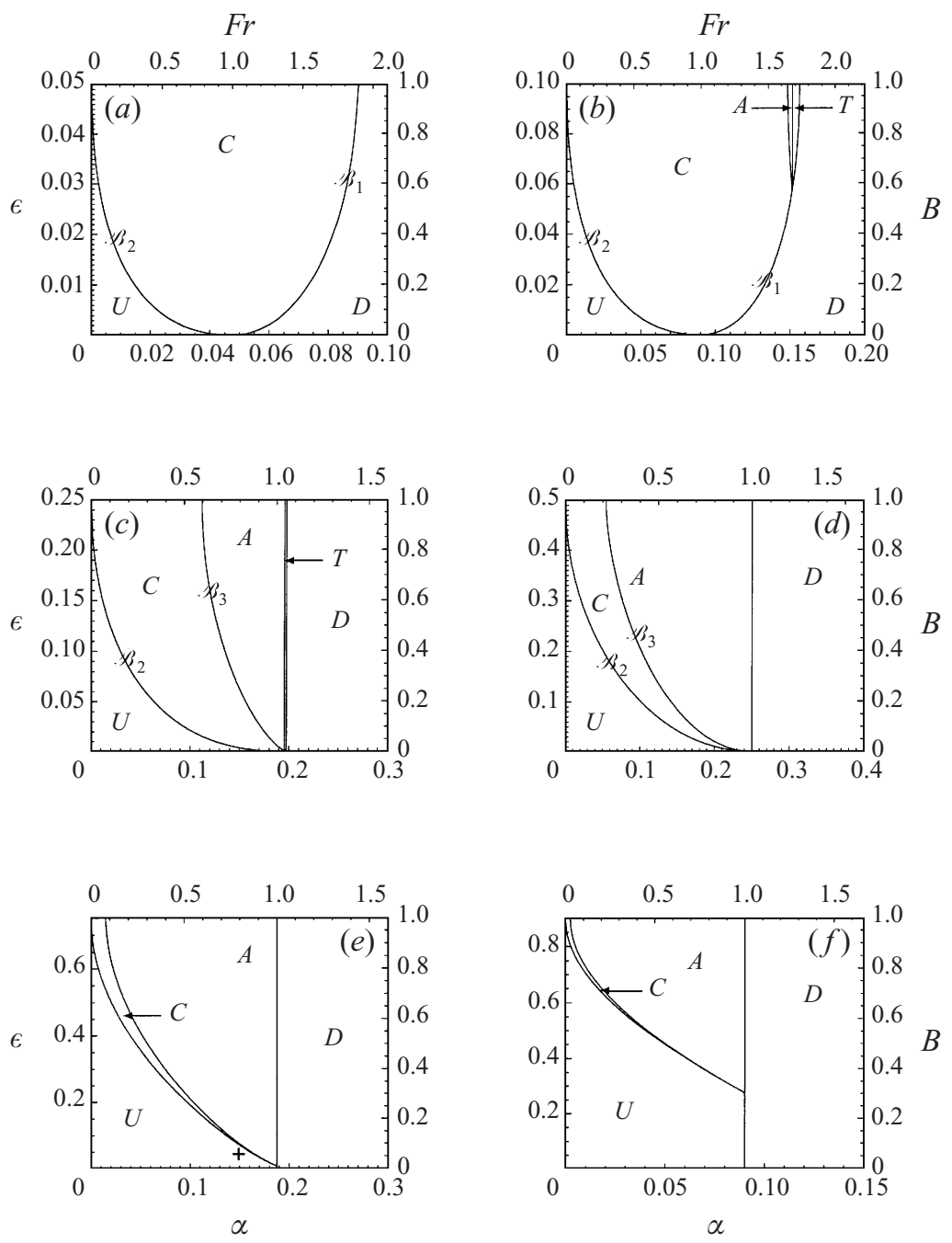

FIGURE 5. Regimes diagrams showing the regions of the $(\alpha, \epsilon)$ parameter space associated with the different types of hydraulic solution of (3.5). (a) $Y_{0}=0.05$, (b) $Y_{0}=0.1$, (c) $Y_{0}=0.25$, (d) $Y_{0}=0.5$, (e) $Y_{0}=0.75$ and $(f) Y_{0}=0.9$. The regimes are $U$ - subcritical (as waves can propagate upstream), $C$ - constriction-controlled (CC), $A$ - approach-controlled (AC), $T$ - twin supercritical transition and $D$ - supercritical (as waves are swept downstream). The curve $\mathscr{B}_{1}$ is the CC/supercritical boundary, $\mathscr{B}_{2}$ the $\mathrm{CC} /$ subcritical boundary, and $\mathscr{B}_{3}$ the AC/CC boundary. The ' + ' in $(e)$ shows the parameter values corresponding to the cusp catastrophe of $\S 4.2$ and figure 8 and the flow evolution of figure 16.

fraction of the shelf blocked by the topographic indentation and so varies from zero to one. In some parameter regimes more than one type of flow is possible. Numerical integrations of the initial value problem for the full dispersive wave equation (2.9) as in $\S 5$ show that, in general, if a steady state with undisturbed upstream conditions, or a flow without an SL, exists then the flow evolves to this state and figure 5 is drawn on this basis. Small dispersion, however, appears to alter the relative stability of solutions in small, exceptional, regions of parameter space and $\$ \$ 4.2,5.3$ discuss how weak dispersion leads to an $\mathrm{AC}$ flow in a region where a well-behaved hydraulic subcritical flow exists. 
Let $Y_{0 C}$ solve simultaneously

$$
\frac{1}{8}\left(1+Y_{0 C}\right)^{2}\left(1-Y_{0 C}\right)=Y(1-Y)^{2}, \quad Y=\frac{1}{3}-\frac{1}{6}\left(4-3\left(1+Y_{0 C}\right)^{2}\right)^{1 / 2},
$$

so $Y_{0 C} \approx 0.09384$. Then the regime diagram takes one of the following five forms.

Type I: $\quad 0<Y_{0}<Y_{0 C}$. Only three hydraulic solutions - supercritical, $\mathrm{CC}$ and subcritical - occur. Figure 5(a) shows the example $Y_{0}=0.05$, with the CC/supercritical and CC/subcritical boundaries the curves $\mathscr{B}_{1}$ and $\mathscr{B}_{2}$, respectively. $\mathscr{B}_{1}$ and $\mathscr{B}_{2}$ meet at $\left(\alpha_{1}, 0\right)$, where $\alpha_{1}=Y_{0}\left(1-Y_{0}\right)$, and, to leading order in $Y_{0}$, the diagram is symmetric about the line $\alpha=\alpha_{1}$.

Type II: $\quad Y_{0 C}<Y_{0}<\frac{1}{9}$. All five hydraulic solutions occur. Figure $5(b)$ shows the example $Y_{0}=0.1$. The new AC and TSL regimes lie above the point where $\mathscr{B}_{1}$ meets the line $\alpha=\frac{1}{8}\left(1+Y_{0}\right)^{2}\left(=\alpha_{2}\right.$, say). The TSL regime lies between $\alpha=\alpha_{2}$ and $\mathscr{B}_{1}$. The CC/AC boundary, $\mathscr{B}_{3}$ (say), begins at the same point with the AC region lying between $\mathscr{B}_{3}$ and $\alpha=\alpha_{2}$. With increasing $Y_{0}$ this multiple intersection moves down the curve $\mathscr{B}_{1}$ towards $\alpha_{1}$.

Type III: $\frac{1}{9}<Y_{0}<\frac{1}{3}$. This type differs from Type II only at the TSL/supercritical boundary. Figure 5(c) shows the example $Y_{0}=0.25$. The TSL/supercritical boundary is now the line $\alpha=4 /\left[27\left(1-Y_{0}\right)\right]\left(=\alpha_{3}\right.$, say) for $\epsilon \geqslant \epsilon_{3}$ (where $\left.0<\epsilon_{3}<Y_{0}\right)$ and $\mathscr{B}_{1}$ otherwise. With increasing $Y_{0}$ the intersections of $\mathscr{B}_{1}$ and the lines $\alpha=\alpha_{2}$ and $\alpha=\alpha_{3}$ move down $\mathscr{B}_{1}$, until for $Y_{0}=\frac{1}{3}$ they both become $\left(\alpha_{1}, 0\right)$ and the TSL region vanishes.

Type IV: $\frac{1}{3}<Y_{0}<\frac{2}{3}$. This type is qualitatively similar to the case $Y_{0}=\frac{1}{2}$ described by $\mathrm{HJH}$ and shown here in figure $5(d)$. The AC/supercritical boundary is $\alpha=\alpha_{1}$ and $\mathscr{B}_{2}$ and $\mathscr{B}_{3}$ intersect this boundary at $\epsilon=0$. Only four hydraulic solutions occur: subcritical, $\mathrm{CC}, \mathrm{AC}$ and supercritical.

Type V: $\frac{2}{3}<Y_{0}<1$. This type has the same four hydraulic solutions and AC/supercritical boundary as Type IV. Figures $5(e)$ and $5(f)$ show the examples $Y_{0}=0.75$ and 0.9. The CC region is always thin. The intersection of the curves $\mathscr{B}_{2}$ and $\mathscr{B}_{3}$ on the line $\alpha=\alpha_{1}$ now occurs in $\epsilon>0$ and moves up the line as $Y_{0}$ increases, reaching $\epsilon=1$ in the limit $Y_{0} \rightarrow 1$, when only supercritical and subcritical flows occur.

\section{Steady dispersive solutions}

Steady, dispersive solutions of (2.9) with $\mu>0$ have been included in figures 3 and 4 for AC and TSL flows. The main effect of dispersion is to smooth sharp changes in the flow: in the $\cos ^{4}$ topography of figure 3 the finite- $\mu$ solutions smooth the solutions near $\chi=1$ where $f^{\text {(iv) }}$ is discontinuous. Here the value of $\mu$ for comparison with the $\mathrm{CD}$ integration of the full equations follows by choosing $\mu$ so that the topography occupies the region $|\chi|<1$. For the CD integration of HJH11(c) this gives $\mu=(\pi / 16)^{2}$ and it can be seen how closely the solution of (2.9) with this value of $\mu$ reproduces the $\mathrm{CD}$ integration near $\chi=1$. The agreement is not as close at the SL once the jump becomes steep as neglected higher-order derivatives become important. However the dispersive solution shows accurately the centre of the jump and where the CD solution begins to deviate from the hydraulic solution. The Newton method of the Appendix is particularly effective for these flows as the form of the flow far upstream and far downstream is known and the flow can be posed as a boundary value problem.

The Newton method also provides rapid solutions for steady completely supercritical flows. No examples are given here since, apart from the smoothing at sharp 

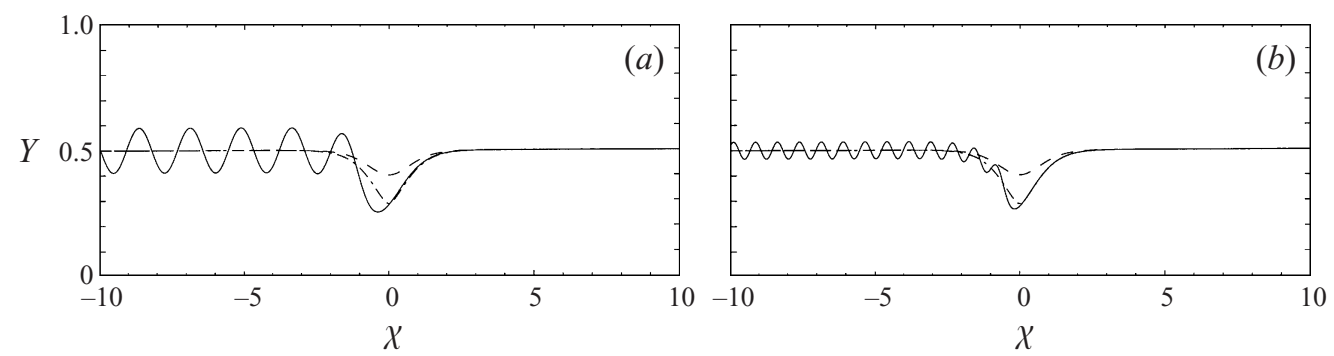

FIGURE 6. The interface $\mathscr{C}$ (solid line) in steady subcritical flow with $Y_{0}=0.5, \alpha=0.1$ and $\epsilon=0.1$, with the shelf-edge (dashed) and the hydraulic $(\mu=0)$ solution (dash-dotted). $(a) \mu=0.5$. (b) $\mu=0.1$. For both values of $\mu$ the standing waves in the lee of the obstacle have approximately the same wavelength relative to the channel width. In $(a)$ this wavelength is closer to the topographic scale and the topography forces the waves more efficiently, giving a wavetrain with amplitude approximately twice the amplitude of the topographic perturbation. In $(b)$ the wavetrain amplitude is less than $\epsilon$.

changes in the topography, as in the supercritical upstream section of figure 3 , the departure of the finite- $\mu$ flows from the hydraulic solutions is imperceptible.

Finite-amplitude wavetrains of the form discussed in CJ can stand in subcritical flows. Requiring that the deviation of the material surface $\mathscr{C}$ remains bounded is not sufficient to give a unique solution as wavetrains can appear in steady solutions upstream of the topography. However, as noted in $\S 3.3$, apart from small exceptional regions of parameter space, time-dependent integrations in the subcritical regime evolve to flows with no disturbance far upstream. Numerically integrating the steady form of (2.9) inwards from undisturbed flow at $\chi=\infty$ as an initial value problem gives a stable problem with a unique solution. Figure 6 shows steady subcritical flow from a Runge-Kutta integration. The waves in the lee of the obstacle for both values of $\mu$ have approximately the same wavelength relative to the channel width and correspond to the periodic free wavetrains of $\mathrm{CJ}$ standing in the oncoming flow of speed $\alpha$. The wavelength in $(a)$ is closer to the topographic scale so the topography forces the waves more efficiently, giving a wavetrain with amplitude approximately twice the amplitude of the topographic perturbation. In $(b)$ the disparity between the wavelength and the topographic length scale is larger and the wavetrain amplitude reduced by over one half.

The remaining flow type is CC flow. As in fully subcritical flow, the flow is subcritical upstream of the topography and so may support a steady upstreamstanding wavetrain. It appears that for any set of parameters there is a continuum of steady solutions with upstream wavetrains of various amplitudes all of which give the same supercritical flow downstream of the control at the constriction. It is unclear how to determine the unique steady solution (if it exists) to a given initial value problem. It might be possible to use nonlinear group-velocity arguments as in Smyth (1987) to determine a unique solution but this will not be pursued further here. The time-dependent integrations of $\S 5$ show that for some parameter values, like those of figure 12, there appears to be little or no upstream wavetrain whilst others, like those of figure 9 , show wavetrains that remain unsteady.

\subsection{The structure of supercritical leaps}

In the steady flows described above, save those with SLs, the displacement of the material interface $\mathscr{C}$ in the limit $\mu \rightarrow 0$ varies slowly for slow topographic variations. In an SL the displacement changes rapidly although the topography changes slowly. The 
(a)

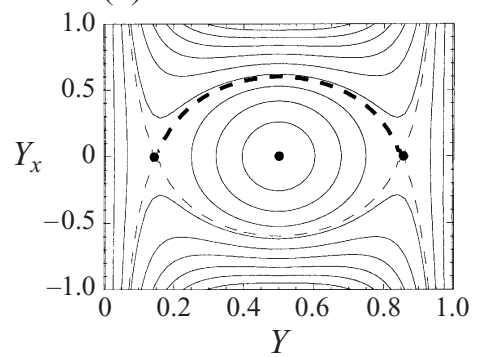

(b)

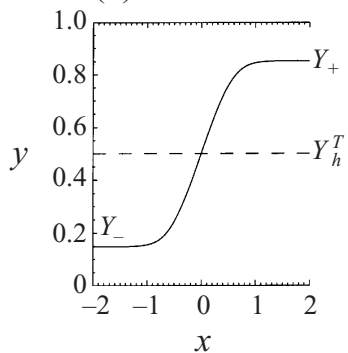

(c)

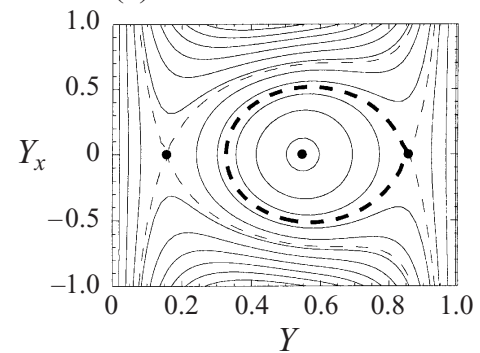

Figure 7. (a) Phase trajectories for equation (4.2). Here $Y_{h}=\frac{1}{2}, \alpha=0.1$ and $\Psi_{0}=0.05$. The insets and outsets of the saddle points are dashed. For these parameter values the two saddle points have the same 'potential' $V$. The heteroclinic orbit joining the saddle points and passing through the region $Y_{x}>0$ is shown dashed in bold. (b) The kink soliton corresponding to the bold trajectory of (a), monotonically joining $Y_{-}$to $Y_{+}$. (c) Phase trajectories for $Y_{h}=0.55, \alpha=0.1$ and $\Psi_{0}=0.055$. The saddle points (filled circles, $\bullet$ ) have different potentials. The homoclinic orbit (bold dashed) from the saddle point at larger $Y$ circles the centre (also a filled circle, $\bullet$ ) at smaller $Y$.

exact form of the leap follows by considering the governing equation (2.9) in a layer, centred on the SL, that is thin compared to the scale of the topography. Equation (2.9) shows that this layer has thickness of order $\mu^{1 / 2} \ll 1$ on the topographic scale $\chi$, and so the relevant interior variable for the SL is $x$. In effect the governing vorticity equation becomes the full equation (2.4) and a steady CD solution should be sought. However, the formal ordering in $\mu$ leading to (2.9) remains valid provided higher-order derivatives of $Y(\chi)$ remain small. From CJ this appears to be so provided the slope of the interface remains moderate on the shelf-width scale $\left(Y_{x} \lesssim 0.5\right.$ in figure 7$)$. The following description of the SL is thus based on (2.9) and the result compared with the CD integration of HJH11(c).

Take the origin for $\chi$ to be at the SL. Then the governing equation inside the SL comes from the steady form of (2.9) as

$$
\Psi_{h}\left(Y, Y_{h}^{T}\right)-\frac{1}{3} Y(1-Y) \frac{\mathrm{d}}{\mathrm{d} x}\left[Y(1-Y) Y_{x}\right]=\Psi_{0}
$$

where $Y_{h}^{T}$ is the (constant, on this scale) topographic displacement at the SL. Solutions of (4.1) are discussed in detail in CJ. Multiplying (4.1) by $Y_{x}$ and integrating with respect to $x$ gives the phase-plane equation

$$
\frac{1}{6} Y^{2}(1-Y)^{2} Y_{x}^{2}+V\left(Y, Y_{h}^{T}\right)=E
$$

where $E$ is an arbitrary constant and the 'potential' $V$ is given by

$$
V\left(Y, Y_{h}\right)=\Psi_{0} Y+\frac{1}{2}\left[-\alpha-Y_{h}+\frac{1}{2} Y_{h}^{2}-\frac{1}{4} Y^{2}+\frac{2}{3} Y\right] Y^{2}-\frac{1}{6}\left(Y-Y_{h}\right)^{3} H\left(Y-Y_{h}\right) \text {. }
$$

In the $\left(Y, Y_{x}\right)$ phase plane maxima of $V$ give saddle points and minima of $V$ give centres. Thus (3.3) shows that centres correspond to subcritical flow and saddle points to supercritical flow. The solutions of (4.1) relevant to an SL are the kink solitons that monotonically join the two heights $Y_{-}$and $Y_{+}$immediately outside the jump, asymptoting exponentially to each limit. These correspond in the phase plane to heteroclinic orbits joining two different saddle points at the same 'potential'. Leaps thus occur only between supercritical flows. Figure 7(a) shows a phase plane portrait for an SL. The relevant heteroclinic orbit is shown bold and dashed. Figure 7(b) shows the corresponding kink soliton solution. Since the potential surface is locally flat at the saddle points and the saddles have the same potential, they satisfy the 
three equations

$$
\begin{aligned}
V_{Y}\left(Y_{-}, Y_{h}^{T}\right) & =0, \\
V_{Y}\left(Y_{+}, Y_{h}^{T}\right) & =0, \\
V\left(Y_{-}, Y_{h}^{T}\right) & =V\left(Y_{+}, Y_{h}^{T}\right) .
\end{aligned}
$$

In terms of the hydraulic function $\Psi_{h}$ these become the SL matching conditions (3.13) and thus support the multiplicative function chosen in moving from (3.11) to (3.12).

Because $\Psi_{Y Y}$ is discontinuous across the step a general explicit solution of (3.13) is not straightforward for AC flows. However the SL in TSL flows does not cross the step and a complete solution is possible. The combination of the requirement that $\Psi_{h}\left(Y, Y_{h}^{T}\right)-\Psi_{0}$, regarded as a cubic in $Y$, vanishes at three points and that the integral between the outer zeros vanishes forces the cubic to be antisymmetric about the middle zero. This middle zero coincides with the inflection point of the cubic which, in the TSL regime (where $Y_{0}<\frac{1}{3}$ ), lies at $Y=\frac{1}{3}$. As the zeros at $Y=Y_{+,-}$ lie symmetrically either side of $Y=\frac{1}{3}$ and the disturbance speed $c$ is even in $Y-\frac{1}{3}$, $c\left(Y_{-}\right)=c\left(Y_{+}\right)$: the speed $c$ of small disturbances to the interface is continuous across the SL when seen on the long length scale $\chi$. Writing $\hat{Y}=Y-\frac{1}{3}$ and $Y_{+,-}=\frac{1}{3} \pm \gamma$ gives

$$
\Psi_{h}\left(Y, Y_{h}^{T}\right)-\Psi_{0}=\frac{1}{3}\left(\hat{Y}^{3}-\gamma^{2} \hat{Y}\right) .
$$

Requiring both sides to be equal at $\hat{Y}=0$ and to have the same slope there gives the two equations

$$
\left(Y_{h}^{T}\right)^{2}=3 \Psi_{0}+\frac{1}{9}-\alpha, \quad \gamma^{2}=\left(Y_{h}^{T}\right)^{2}+\frac{1}{3}-2 \alpha .
$$

For a material contour originating from $\left(Y_{0}, Y_{0}\right)$ as in TSL flows, the streamfunction constant is $\Psi_{0}=\alpha Y_{0}$. Substituting into (4.6) and rearranging gives

$$
Y_{+,-}=\frac{1}{3} \pm\left[\frac{4}{9}-3 \alpha\left(1-Y_{0}\right)\right]^{1 / 2}, \quad Y_{h}^{T}=\left[\frac{1}{9}-\alpha\left(1-3 Y_{0}\right)\right]^{1 / 2},
$$

the explicit solution for the three unknowns $Y_{+}, Y_{-}$and $Y_{h}^{T}$. Equation (4.2) can be written

with solution

$$
\frac{4}{3} Y^{2}(1-Y)^{2} Y_{x}^{2}=(\hat{Y}+\gamma)^{2}(\hat{Y}-\gamma)^{2},
$$

$\frac{1}{2} \sqrt{3}\left(x-x_{0}\right)=Y+\frac{1}{2 \gamma}\left(\gamma^{2}-\frac{1}{3} \gamma-\frac{2}{9}\right) \log \left(Y_{+}-Y\right)-\frac{1}{2 \gamma}\left(\gamma^{2}+\frac{1}{3} \gamma-\frac{2}{9}\right) \log \left(Y-Y_{-}\right)$,

where $x_{0}$ is an arbitrary constant of translation. This is the explicit form for the interface displacement within the SL. As expected, $x \rightarrow \pm \infty$ as $Y \rightarrow Y_{+,-}$and $x=x_{0}$ when $Y=\frac{1}{3}$.

Since the disturbance speed $c$ is continuous across the SL of a TSL the momentum $c \delta$ is conserved across the SL (taking the mass $\delta$ as conserved). This contrasts with the SL of AC flow where $c$ is not continuous across the SL. Crossing the step in AC flow appears to apply an impulse to the interface that alters the momentum of disturbances on the interface. For the non-rotating stratified flow of Lawrence (1993) and Baines (1984), the material interface cannot pass through the ridge and so the SL would appear to be more closely modelled by the continuous-disturbance-speed SLs of the TSL regime. This leads to the hypothesis that the criterion fixing the position and size of SLs in two-layer stratified flows is the requirement that both the 


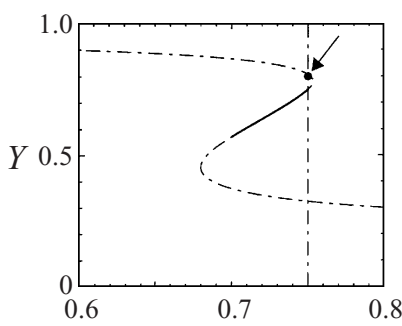

(a)

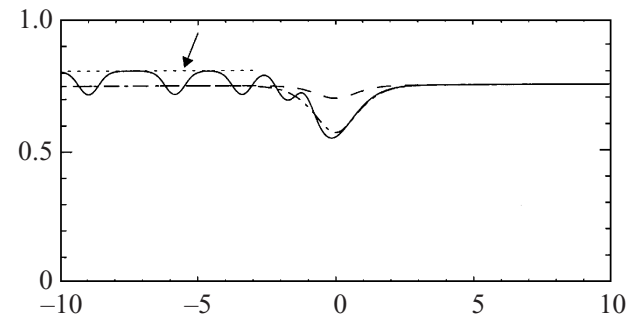

(b)
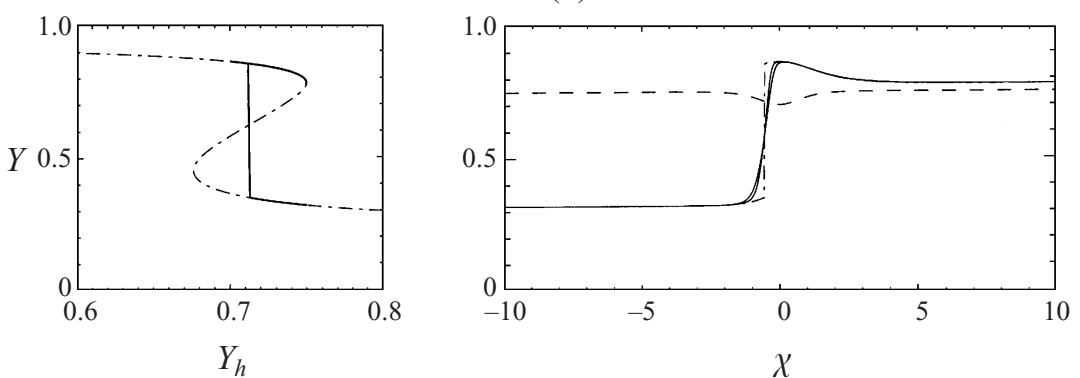

FiguRE 8. Steady flows for $Y_{0}=0.75, \epsilon=0.05$ and $\alpha=0.15$, corresponding to ' + ' in figure $5(e)$. The left-hand diagram in each part is the hydraulic diagram for the flow. The basic solution $\Psi_{h}$ is shown as dash-dotted and is the same in each part. The relevant hydraulic solution constructed from $\Psi_{h}$ is shown in bold on each hydraulic diagram. The right-hand figures show the dispersive solutions (non-zero $\mu$ ) (solid lines) with the topography (dashed) and the relevant hydraulic solution (dash-dotted). (a) Subcritical flow for $\mu=0.15443$. The inaccessible supercritical solution is indicated by an arrow in each part. On the hydraulic diagram it is a single point (filled circle) and in the channel it gives the undisturbed level for the lee wavetrain (dotted). For this extreme value of $\mu$ the lee waves are periodic almost-solitary waves. (b) AC flow for $\mu=0.1,0.2$. The solutions change little from the hydraulic solution, simply becoming smoother with increasing $\mu$. For $\mu<0.15443$ the subcritical and AC flows coexist but for larger $\mu$ the subcritical solution disappears.

streamfunction on the interface and the long-wave speed of small disturbances to the interface are the same on both sides of the leap.

\subsection{Loss of the subcritical solution branch}

Time-dependent integrations, outside small exceptional regions, show flows in the subcritical regime evolving to solutions undisturbed far upstream $\left(Y \rightarrow Y_{0}\right.$ as $\left.\chi \rightarrow \infty\right)$ and thus the constant streamfunction value on the interface $\mathscr{C}$ is fixed as $\Psi_{0}=\alpha Y_{0}$ in subcritical flows. Further, the periodic wavetrain of a steady subcritical flow lies downstream of the topographic perturbation where the shelf width has regained its upstream value, $Y_{0}$. Thus in the wavetrain $Y_{h}$ is fixed and equal to $Y_{0}$. These conditions differ from those for an SL in $\S 4.1$ since the SL occurs at the perturbation (where $Y_{h}<Y_{0}$ ) and $\Psi_{0} \neq \alpha Y_{0}$ determines the upstream influence in AC flow (although, as noted in $\S 4.1, \Psi_{0}=\alpha Y_{0}$ in TSL flows, where the upstream flow is undisturbed). Figure 7(c) shows a typical phase portrait for subcritical flow, with $\alpha=0.1, Y_{0}=0.55$ and so $\Psi_{0}=0.055$. The two saddle points have different potentials and so no leap is possible. A homoclinic orbit from one saddle point (a supercritical solution) circles the centre, giving a negative-amplitude solitary wave with far-field displacement equal to the supercritical solution. The constant downstream displacement of the hydraulic $(\mu=0)$ subcritical solution is given by $Y=Y_{0}$ at the centre. The downstream 




(a)

(b)

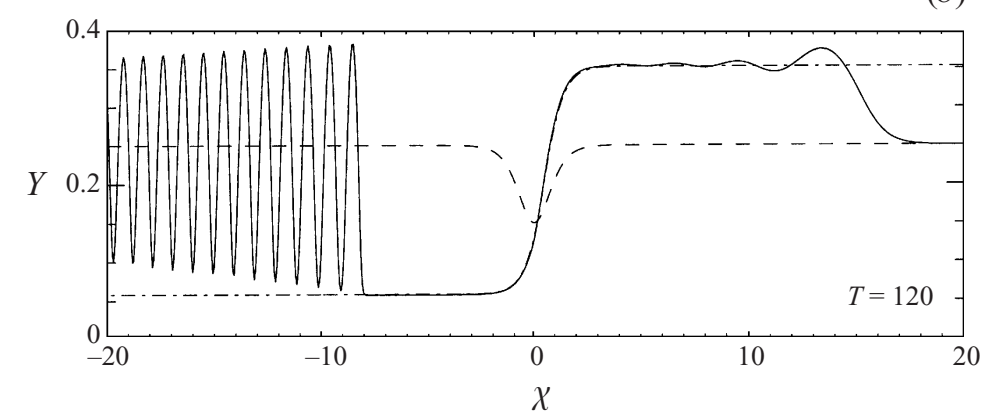

FIGURE 9. A CC solution of (2.9) and (2.11) for $Y_{0}=0.25, \epsilon=0.1, \alpha=0.1$ and $\mu=0.2$. (a) The evolution of $A=Y-Y_{0}$. (b) The interface $\mathscr{C}$ at $T=120$ with the shelf-edge (dashed) and the hydraulic solution (dash-dotted).

wavetrains of steady subcritical flows with $\mu>0$ are given by the orbits circling the centre. Increasing $\mu$ (so decreasing the disparity between the wavelength and the topographic scale and generating waves more efficiently, as in figure 9) increases the radius of the corresponding orbit. In some small regions of parameter space, when $\mu$ is increased past some critical value $\mu_{0}$ (the topography is sufficiently short) the required amplitude of the forced wavetrain corresponds to an orbit radius that exceeds the radius determined by the solitary-wave separatrix and the subcritical solution branch disappears. The critical value $\mu_{0}$ is determined by noting that the 'potential' $V$ defines a potential well about $Y=Y_{0}$ and the loss of the subcritical solution branch corresponds to the escape of a particle from this well. The 'energy' of a particle is the value, immediately after the topographic perturbation, of $E$ given by (4.2). For any $\mu$ it follows by integrating the steady form of (2.9) inwards from $x \gg 1$, as in figure 6 . The critical value $\mu_{0}$ is the value of $\mu$ for which the energy $E$ equals the energy on the separatrix, $E_{0}$ (say), i.e. the potential $V$ at the saddle point. For $\mu>\mu_{0}, E$ exceeds $E_{0}$ and the particle escapes.

In the present geometry subcritical solution loss occurs when the $\mathrm{CC}$ region of parameter space is very narrow and the $\mathrm{AC}$ and subcritical regions almost border each other. Figure 5 shows that this is possible for particular values of $\alpha$ and $\epsilon$ when $Y_{0}>2 / 3$. Figure 8 gives steady flows for $Y_{0}=0.75, \epsilon=0.05$ and $\alpha=0.15$, indicated by ' + ' on figure 5(e), where the CC region is narrow. An hydraulic $(\mu=0)$ subcritical 
flow exists. For $\mu>0$ a downstream wavetrain appears and grows in amplitude with increasing $\mu$. At the critical value $\mu_{0} \approx 0.15444$, the wavetrain has grown sufficiently to correspond to the solitary wave forming the separatrix in the phase plane. For $\mu<\mu_{0}$ subcritical flows exist and for $\mu>\mu_{0}$ subcritical solutions disappear. Figure 8(a) shows subcritical flow at $\mu=0.15443$ just before this branch of solutions vanishes. The hydraulic diagram shows the $(\mu=0)$ subcritical solution in bold, with an arrow showing the supercritical solution corresponding to the solitary-wave saddle point. This supercritical solution is not smoothly accessible from the subcritical solution as this would require passing into $Y_{h}>Y_{0}$ and, by (2.11), $Y_{h} \leqslant Y_{0}$ always. The interface $\mathscr{C}$ in figure 8(a) shows that the wavetrain has become a train of almost-solitary waves with broad crests, narrow troughs and undisturbed level given by the 'inaccessible' supercritical $(\mu=0)$ solution. For $\mu>\mu_{0}$ the sole candidate for steady flow is the AC flow of figure $8(b)$, containing an SL with both $Y_{h}$ and $\Psi_{0}$ differing from subcritical flow. The hydraulic diagram shows AC flow for $\mu=0$ when it coexists with subcritical flow. The right-hand part of figure $8(b)$ gives the interface $\mathscr{C}$ for $\mu=0,0.1$ and 0.2 (so $\mu>\mu_{0}$ ) showing that this branch of solutions varies smoothly with $\mu$, simply becoming slightly smoother as dispersion increases. The flow exhibits a cusp catastrophe under the two control parameters $Y_{h}$ and $\Psi_{0}$ as the strength of the perturbation is increased, dropping from the metastable subcritical flow to an absolutely stable AC flow with different values of both $Y_{h}$ and $\Psi_{0}$ (Thompson \& Stewart 1986; Jackson 1990).

\section{Unsteady dispersive solutions}

This section presents numerical integrations of the initial value problem (2.9) with topography (2.11), obtained using pseudospectral operators in space and a third-order Runge-Kutta integration in time. The method is an adaption of that proposed for weakly nonlinear dispersive systems by Fornberg \& Whitham (1978). The accuracy and other details of these spectral methods are discussed in Canuto et al. (1988). Three different values of $Y_{0}$ are considered for various $\epsilon$ and $\alpha$ with $\mu=0.2$ everywhere except in the final figure, figure 16 , where $\mu=0.05$

$$
\text { 5.1. } Y_{0}=0.25
$$

The hydraulic diagram for $Y_{0}=0.25$ shows that five types of hydraulic flow are possible. Three of these, spanning the CC, AC and TSL regimes, are described here for $\epsilon=0.1$.

Figure 9 shows flow development when $\alpha=0.1$ which falls in the CC regime close to the AC boundary. The initial disturbance forms into two shocks, one propagating upstream and the second forming downstream of the topography. Between these two shocks a CC solution forms, closely described by the hydraulic solution. The upstream shock disperses into an undular bore which appears to remain attached to the topography. The downstream shock forms an undular bore which propagates away leaving the constant-amplitude negative $\left(Y<Y_{0}\right)$ plateau of the hydraulic solution. At large times the solution becomes steady immediately downstream of the topography, but small-amplitude waves are continually generated at the leading edge of the topography to propagate upstream on the positive $\left(Y>Y_{0}\right)$ plateau there. The development of flows within the $\mathrm{CC}$ region, but closer to the subcritical boundary, differs slightly from figure 9. Integrations for $\alpha=0.05$ (not shown here) show the same initial formation of upstream and downstream shocks. However, the upstream bore disperses into a single solitary wave that propagates away to leave a steady upstream plateau, whilst the downstream wavetrain becomes steady and remains 


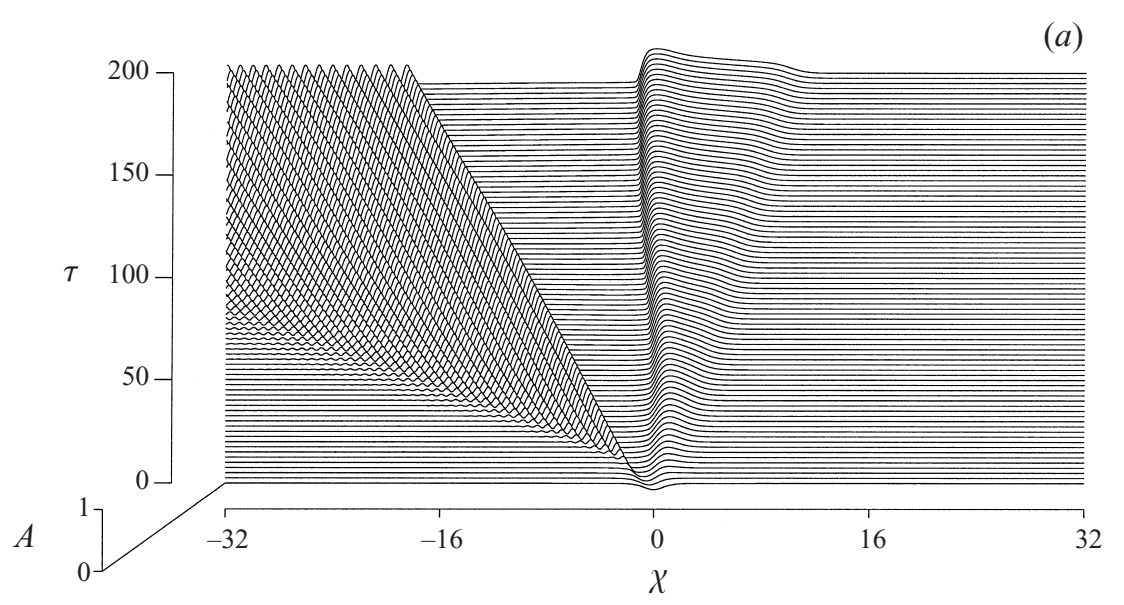

(a)

(b)

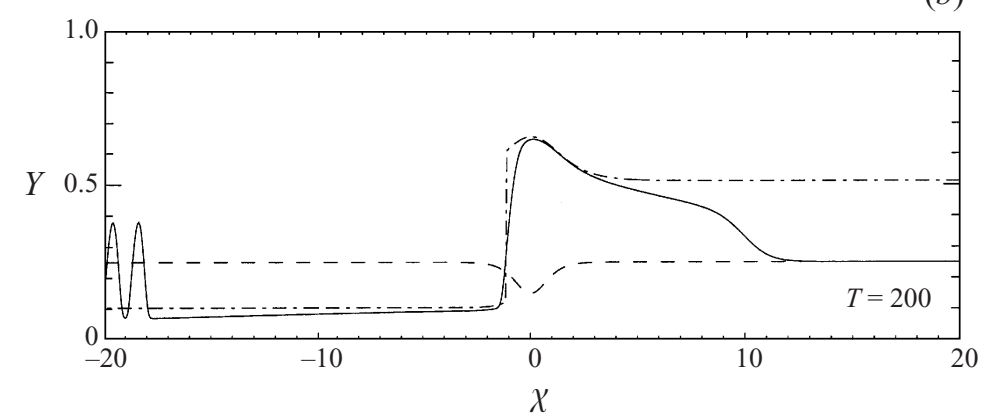

Figure 10. As figure 9 but for an AC solution of (2.9) and (2.11) for $\alpha=0.15$.

attached to the topography (as seen later in figure 12 for $Y_{0}=0.5$ ). The contrast between these finite-amplitude evolutions is similar to that described by Grimshaw \& Smyth (1986) and Smyth (1987) in studying resonant weakly nonlinear solutions of the forced KdV equation. They found for 'positive' forcing that the asymptotic solutions took one of two forms: either a steady positive plateau formed upstream with a steady nonlinear wavetrain downstream, or a steady negative plateau formed downstream with unsteady large-amplitude waves being formed at the leading edge of the topography.

Figure 10 shows AC flow. First a CC-like solution forms at the topography. Then its associated transition steepens and moves to the downstream side of the topography, becoming at large times an SL. The same evolution occurs in the CD example of $\mathrm{AC}$ flow in HJH11. The initial upstream shock of the CC solution evolves into a dispersionless bore with a trailing rarefaction, rather than an undular bore of figure 9. The downstream undular bore propagates rapidly away, leaving behind a negative plateau whose amplitude varies slowly as the flow evolves from CC to AC. Near the topography the solution agrees closely with the hydraulic solution, suggesting that the flow there has almost reached a steady state. At large time the positive upstream plateau and negative downstream plateau move monotonically towards the predicted hydraulic solution, although as figure 10 and the simulations of HJH show, AC flows take far longer to reach an asymptotic state than $\mathrm{CC}$ flows. In contrast to figure 9, dispersion has little effect here apart from smoothing the SL. 
(a)

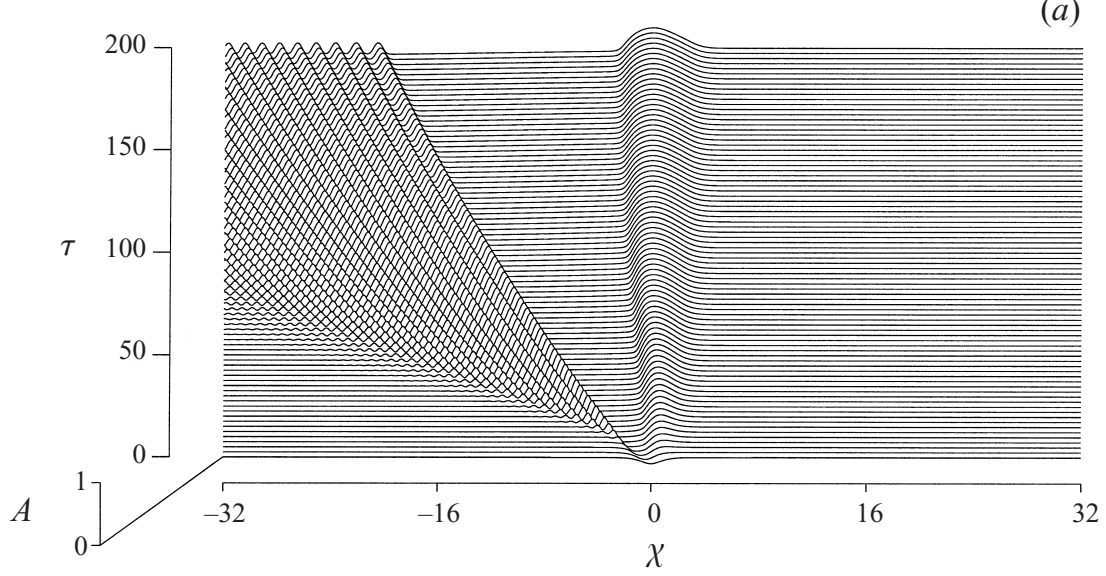

(b)

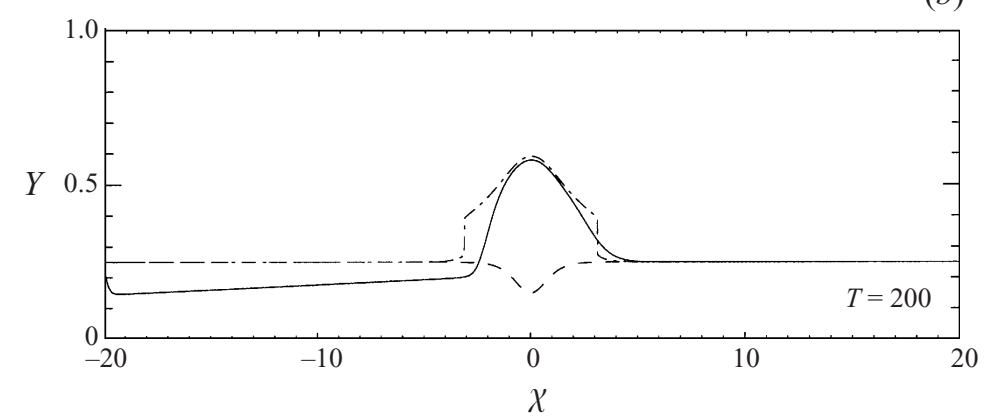

FIGURE 11. As figure 9 but for a TSL solution of (2.9) and (2.11) for $\alpha=0.196$.

Figure 11 shows an evolution from the very narrow TSL regime. The development is typical of supercritical solutions, with no upstream influence and a wavetrain swept downstream. At large time the interface near the topography moves monotonically towards the predicted hydraulic solution, although, as in figure 10, the approach is slow. Once again, dispersion has little effect apart from smoothing the SLs.

$$
\text { 5.2. } Y_{0}=0.5
$$

For $Y_{0}=0.5$, the symmetric, mid-channel-shelf geometry of $\mathrm{HJH}$, the $\mathrm{CC}$ and $\mathrm{AC}$ regimes are of particular interest. This subsection gives examples for the relatively large-amplitude perturbation of $\epsilon=0.25$, the value, in the present notation, used in the $\mathrm{CD}$ simulations of $\mathrm{CC}$ and $\mathrm{AC}$ flows in $\mathrm{HJH}$. For moderate $\epsilon$ flows evolve in a similar, but less extreme, manner.

Figure 12 for $\alpha=0.05$ falls into the CC regime near the subcritical boundary. The flow develops initially like the CC flow of figure 9. However, as the flow lies near the subcritical rather than the AC boundary the subsequent behaviour differs. The downstream undular bore evolves into a stationary lee wavetrain attached to the obstacle and upstream small waves continually generated at the obstacle propagate away along the positive plateau given by the hydraulic solution. The hydraulic solution accurately models the behaviour near the maximum topographic perturbation, but fails due to dispersive effects both upstream and downstream. This solution should be compared with HJH6(a) where $\alpha \approx 0.053$ and $\mu \approx 0.15$. Figure HJH6(a) shows no upstream wave generation as the domain shown is too narrow, but HJH6(b), 


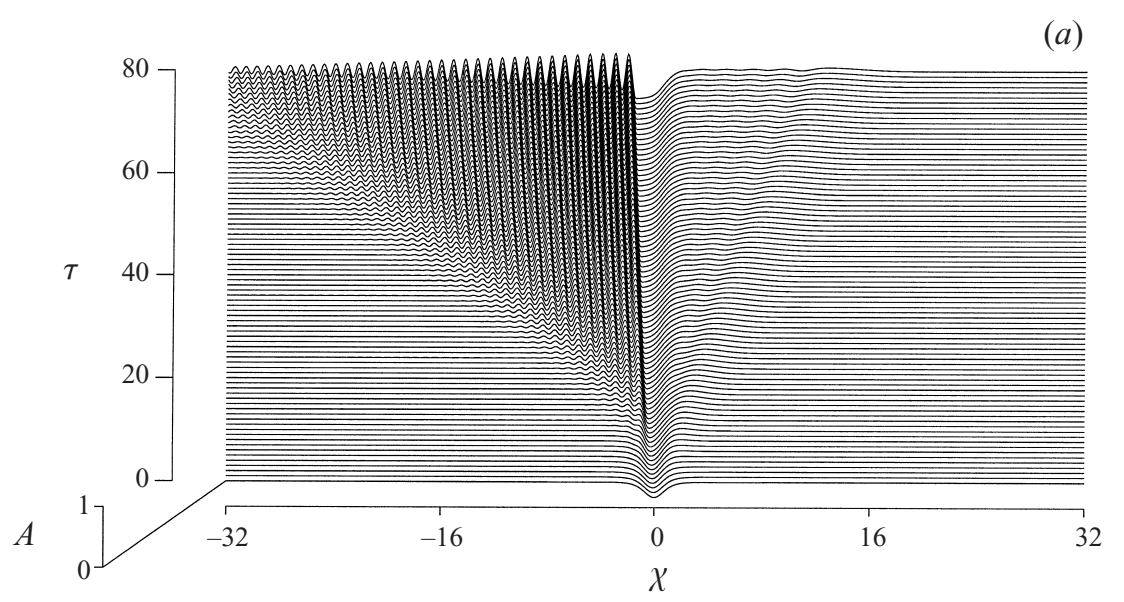

(a)

(b)

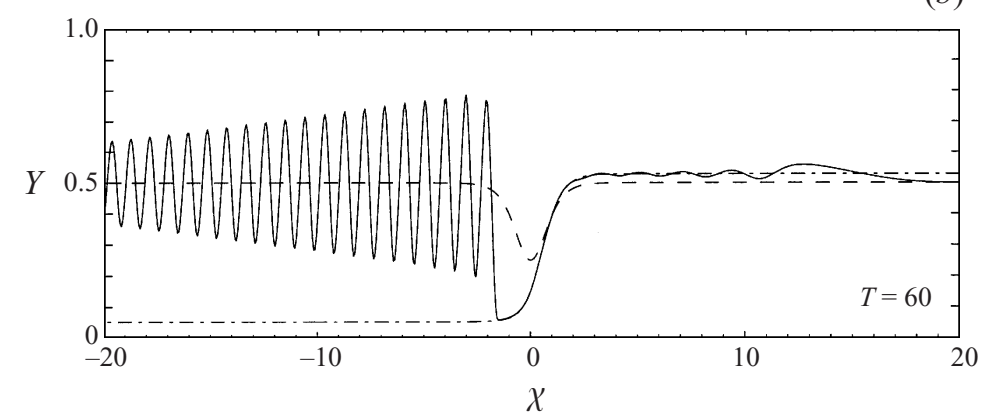

Figure 12. As figure 9 but for a CC solution of (2.9) and (2.11) for $Y_{0}=0.5, \epsilon=0.25, \alpha=0.05$.

where $\mu \approx 1$, does show weak unsteady waves at the leading edge of the topography. In both flows of HJH6 the leading wave of the downstream wavetrain overturns to form an eddy, although for the longer topography this is only a slight pinching. The present formulation requires $Y$ to be single-valued. Dispersive effects in the governing equation $(2.9 a)$ prevent the interface $\mathscr{C}$ from becoming vertical and so, provided the resolution of the computation is sufficiently high, flow evolutions can be followed until $\mathscr{C}$ approaches the vertical. In figure 12 the slope does not become vertical anywhere even though the interfaces of HJH6 show pinching. Once $\mathscr{C}$ approaches the vertical, the closeness of the long-wave integration to the CD simulation is not assured, however, as higher-order derivatives, neglected in the derivation of (2.9a) may become important. A brief discussion and comparison of long-wave and overturning CD integrations for the coastal geometry of Clarke \& Johnson $(1997 a, b)$ is given in Johnson \& Clarke (1998).

Figures 13 and 14 give evolutions in the $\mathrm{AC}$ regime where the $\mathrm{CD}$ integrations of HJH overturn even for long obstacles. Figure 13 with $\alpha=0.1$ corresponds to HJH11 and figure 3 where $\alpha \approx 0.106$ and $\mu \approx 0.04$. The evolution terminates just after $T=65$ when the interface touches, and then attempts to pass through, the wall at $y=0$. Figure 13(b) shows that the touching is at the base of the transition that has moved downstream from its initial position upstream of the topographic maximum. This agrees with HJH11(b) where the transition overturns as it moves downstream of the topographic maximum. The removal of the possibility of overturning leads 


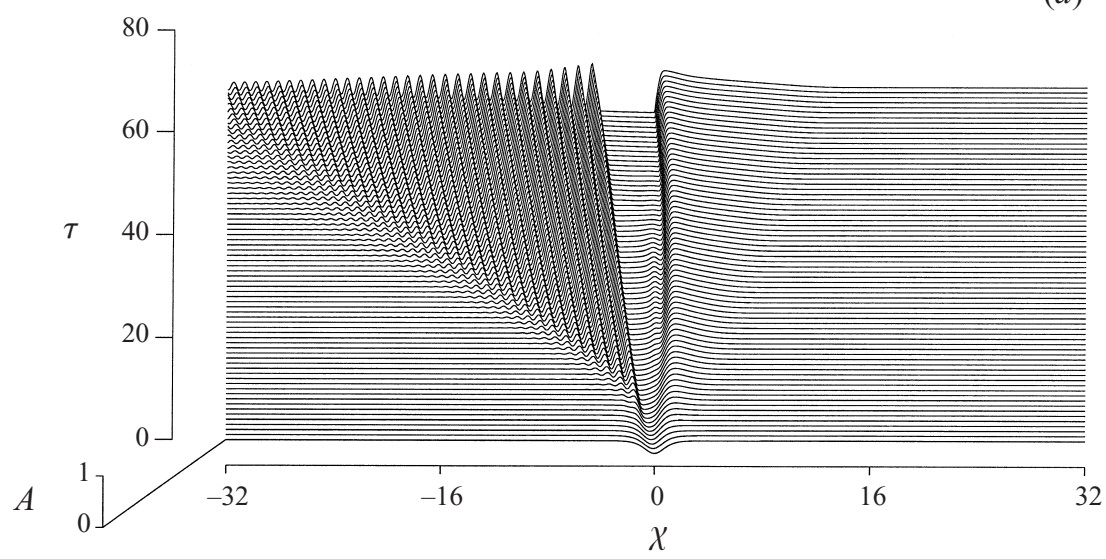

(b)

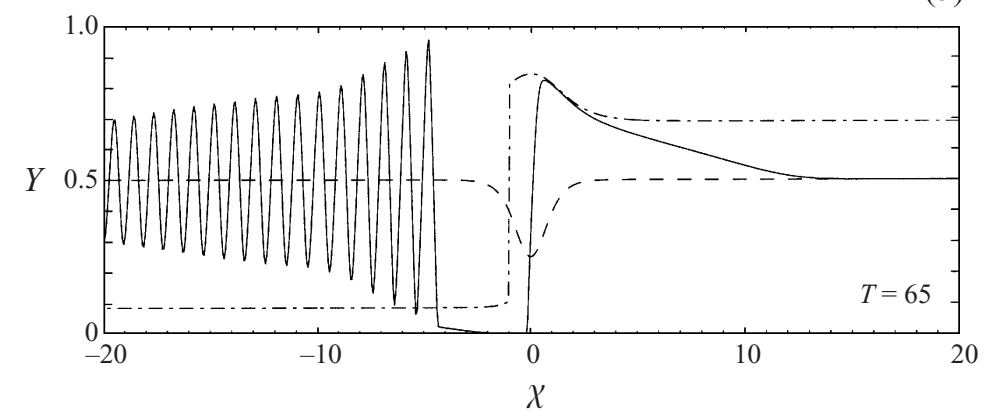

FiguRE 13. As figure 12 but for an AC solution of (2.9) and (2.11) for $\alpha=0.1$. The integration terminates just after $T=65$ as the interface strikes the wall.

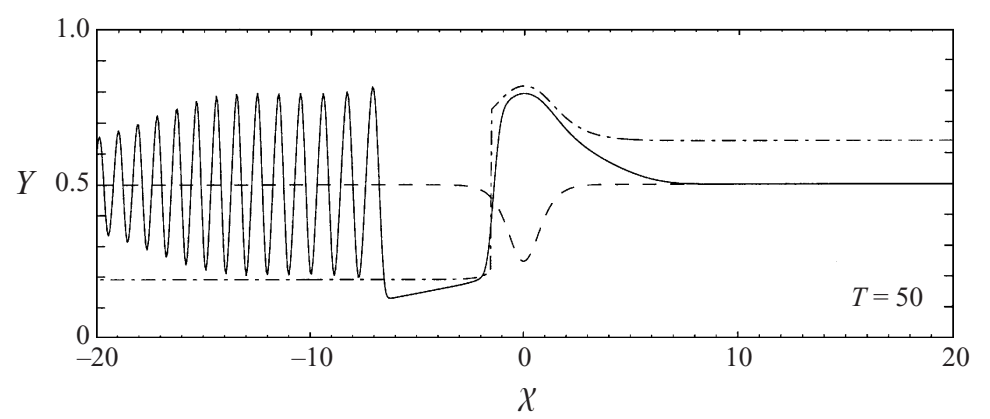

Figure 14. The interface $\mathscr{C}$ at time $T=50$ of an AC solution of (2.9) and (2.11) with $Y_{0}=0.5$, $\epsilon=0.25, \alpha=0.175$ and $\mu=0.2$. The shelf edge is dashed and the hydraulic solution dash-dotted.

the evolution to fail by predicting that the interface passes through the wall. Again, similar behaviour for coastal flows has been noted in Clarke \& Johnson (1997b) and Johnson \& Clarke (1998). Figure 13(a) shows that between $T=20$ and $T=40$ a small wave, possibly a shock or hydraulic jump, forms on the negative plateau immediately downstream of the transition as the solution changes from being CC to $\mathrm{AC}$ flow. The wave moves downstream ahead of the transition, decreasing in amplitude. An identical feature is apparent in the CD simulation of HJH11(a).

Figure 14 shows the interface $\mathscr{C}$ at large time from a second large-amplitude AC 


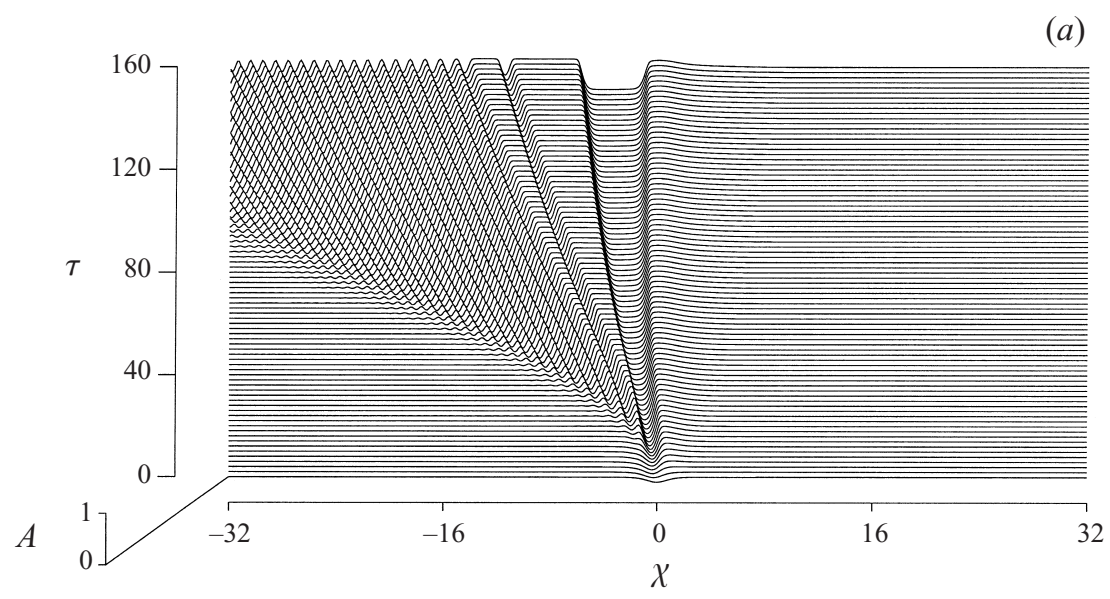

(a)

(b)

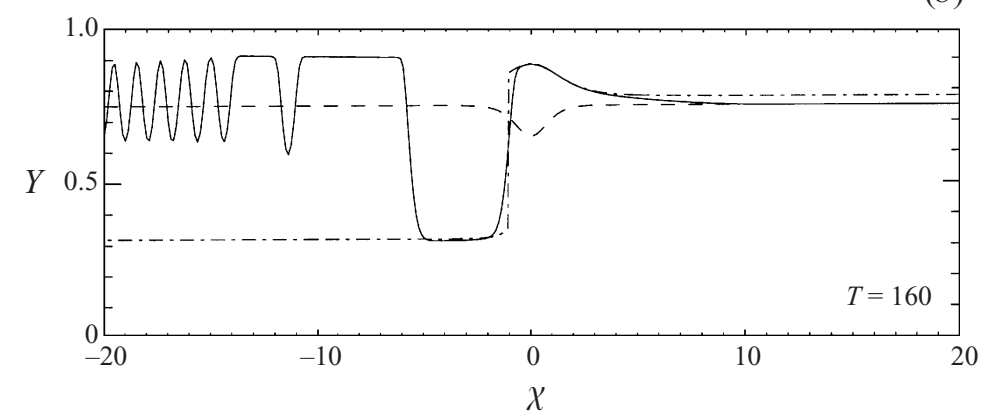

FIGURE 15. As figure 13 but for an AC solution of (2.9) and (2.11) for $Y_{0}=0.75, \epsilon=0.1, \alpha=0.15$ (so $F r=0.8$ ).

flow. A constant-amplitude wake propagates downstream leaving behind a region where the flow evolves monotonically towards the predicted hydraulic solution. This differs from weakly nonlinear solutions where the bore amplitude decreases downstream and also from the CD integrations of $\mathrm{HJH}$ where the wave at the upstream edge of the downstream-propagating bore overturns. As in the evolution of figure 12 , provided the resolution is sufficiently high, integrations of equation (2.9a) do not overturn even when the CD integrations do. The filtering out of short-scale effects in the long-wave equation leads to faster dispersion of the downstream undular bore and prevents overturning of the wave forming the bore's upstream edge.

$$
\text { 5.3. } Y_{0}=0.75
$$

The regime diagram shows four hydraulic solutions for $Y_{0}=0.75$ : subcritical, $\mathrm{CC}$, $\mathrm{AC}$ and supercritical. Except for large $\epsilon$, where the interface overturns early in the evolution, the $\mathrm{CC}$ regime is narrow and so $\mathrm{CC}$ flows for this value of $Y_{0}$ are unlikely to be observed. This leaves subcritical and AC flows as those whose behaviour is of most interest. Two examples are given here.

Figure 15 shows $\mathrm{AC}$ flow with $\epsilon=0.1$ and $\alpha=0.15$ (so $F r=0.8$ ). Upstream the evolution is similar to the other AC flows with a rarefaction propagating away from the topography. The downstream-propagating wavetrain evolves differently. Instead of developing into a solitary wave the leading edge of the downstream undular bore forms into a dispersionless shock which propagates downstream. The shock is a 


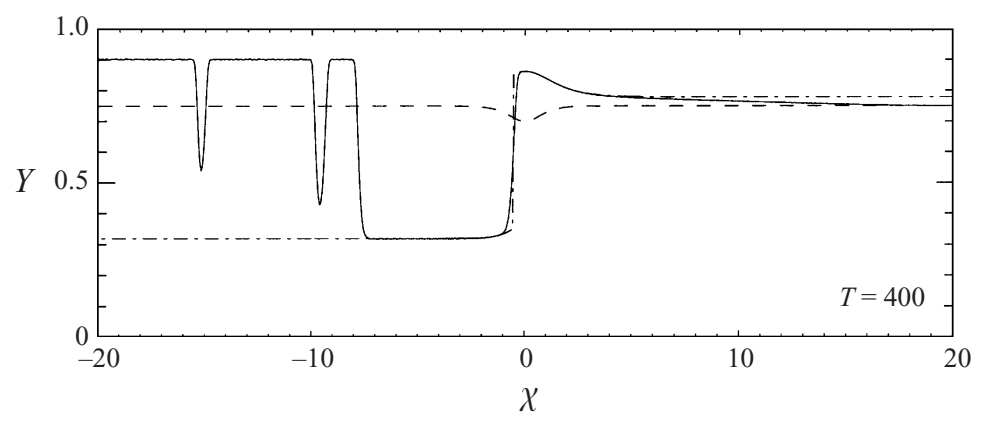

FIGURE 16. An unsteady solution of (2.9) and (2.11) for $Y_{0}=0.75, \epsilon=0.05, \alpha=0.15$ (so $F r=0.8$ ) and $\mu=0.05$, corresponding to the steady flows shown in figure 8 . The interface $\mathscr{C}$ is shown at $T=400$ with the shelf-edge (dashed) and the AC hydraulic solution (dash-dotted).

propagating kink soliton as described in CJ and $\S 4.1$. Here however $Y_{-}$and $Y_{+}$are fixed as the $Y$-positions of the two possible supercritical hydraulic solutions (saddle points) and $Y_{h}^{T}=Y_{0}$. The conditions (3.13) or (4.4) now determine the propagation speed of the shock. Downstream from the shock the wake is beginning to disperse into a train of negative solitary waves of the same form as those given by the dashed trajectory of figure $7(c)$, pointing towards the centre of the channel from the positive plateau of the supercritical solution. At large times the flow near the topographic perturbation becomes steady, with a constant-amplitude positive plateau upstream and negative plateau downstream, in close agreement with the hydraulic solution.

Figure 16 shows the interface $\mathscr{C}$ at large time for a smaller-amplitude perturbation at the same speed $\alpha$ but with much reduced dispersion of $\mu=0.05$. The parameter values correspond to the ' + ' of figure $5(e)$, falling into the subcritical region but close to the AC region. This is the point noted in $\S 4.2$ where the subcritical solution branch disappears when $\mu$ exceeds $\mu_{0} \approx 0.15444$. The dispersion in figure 16 is however far smaller with $\mu<\frac{1}{3} \mu_{0}$. Nevertheless, the solution ignores the well-behaved (metastable) subcritical flow (which has a far smaller-amplitude wavetrain than figure $8(a)$ ), evolving to the coexisting (absolutely stable) AC flow. The evolution is slow because dispersion is weak, but by $T=400$ the interface shows the downstream wavetrain dispersing into a train of negative solitary waves dipping down from the reference level given by the 'inaccessible' supercritical solution, as in figure 15. It appears that when the $\mathrm{CC}$ region of parameter space is too small to offer a barrier between subcritical and $\mathrm{AC}$ regions, unsteady flows can evolve to be $\mathrm{AC}$ well before the topography is so short that the solution passes through the cusp catastrophe of $\S 4.2$.

\section{Discussion}

The simple model introduced in HJH of Rossby-wave hydraulics in a rotating channel has been extended to incorporate higher-order, dispersive, effects. Retaining higher-order terms in the aspect ratio $\mu^{1 / 2}$ of the motion gives a finite-amplitude evolution equation for the potential vorticity interface, which at leading order is the hydraulic equation derived in $\mathrm{HJH}$. At the next order dispersion becomes important, preventing the formation of the shock-like discontinuities typical of hydraulic solutions. The evolution of the interface can thus be efficiently followed up to large time and steady equations integrated to give continuous stationary solutions.

The hydraulic behaviour of the system for simple contractions (only one extremum) 
of the shelf width depends on three non-dimensional parameters: $Y_{0}$, the unperturbed position of the topographic step; $\epsilon$, the maximum perturbation to the position of the step; and $\alpha$, the oncoming flow speed. Here the analysis of HJH for the special symmetric case of $Y_{0}=\frac{1}{2}$ has been extended to the full range of these parameters. Five types of hydraulic solutions occur: subcritical, supercritical, controlled at the constriction (CC), controlled at the leading edge (approach-controlled, AC) and twin supercritical leaps (TSLs). The first four have been described in $\mathrm{HJH}$ but the last occurs only for $Y_{0}<\frac{1}{3}$. For each value of $Y_{0}$ the regimes for these solutions have been delineated in the $(\alpha, \epsilon)$-plane, giving five characteristic types of regime diagrams. The general form depends on whether $0<Y_{0}<\frac{1}{3}, \frac{1}{3}<Y_{0}<\frac{2}{3}$ or $\frac{2}{3}<Y_{0}<1$.

Three of the hydraulic solutions - subcritical, supercritical and CC - are smoothly varying. The remaining two, AC and TSLs, involve sudden jumps of the hydraulic solution between different supercritical branches. Integrations and analysis of the steady form of the finite-amplitude evolution equation show that in the hydraulic limit these supercritical leaps (SLs) correspond to the kink soliton solutions of the unforced problem. Transitions occur between points on the hydraulic curve having the same value of the 'potential' $V$ of (4.3). Hydraulic theory augmented by the jump conditions (3.13) or, equivalently, (4.4) determines the position of an SL. Within the SL dispersive effects are important at leading order. Although the analysis of SLs and TSLs is particularly straightforward for the present model with its simple two-valued potential vorticity distribution, these phenomena should be generic for systems with similarly shaped hydraulic diagrams. The simple single-step shelf discussed here is equivalent to filtering out the higher cross-channel modes present over smoother depth changes and retaining only the fundamental mode. In coastal wave-scattering problems over finite-amplitude topography restricting attention to the fundamental mode has been shown to give accurate approximations to the full multi-mode solutions (Johnson 1990, 1993). Preliminary calculations indicate that the flow behaviours described here are also present in continuously stratified flows.

In supercritical, AC and TSL flows dispersion simply smooths the hydraulic solution where the interface slope would otherwise change rapidly. In subcritical flows a finiteamplitude standing lee wavetrain forms downstream, and upstream the interface displacement is given by the constant value of the hydraulic solution. CC flows show two distinct behaviours depending on whether they lie nearer the subcritical or AC boundaries of the $\mathrm{CC}$ regime. Those near subcritical flow show the constant upstream interface displacement and steady downstream wake typical of subcritical flow. Those near AC flow show the steady downstream displacement of supercritical solutions but at the leading edge of the obstacle show continual, unsteady, generation of waves which propagate upstream superposed on the plateau of the hydraulic solution. In general, all these flows, at least near the perturbation and often elsewhere, are welldescribed by hydraulic theory. However, in some flows for $Y_{0}>\frac{2}{3}$ even very weak dispersion changes the whole character of the flow. This happens when the $\mathrm{CC}$ region separating the subcritical and $\mathrm{AC}$ regions is thin as in figure 5(e) or the $\mathrm{CC}$ and $\mathrm{AC}$ regions are absent and subcritical borders supercritical as in figure $5(f)$. Near these boundaries the steady subcritical solution disappears as the dispersion $\mu$ is increased (the topographic scale reduced) past some critical value $\mu_{0}$, leaving an AC solution as the sole candidate for steady flow. Integrations of the initial value problem show that in these parameter regimes even far smaller dissipation $\left(\mu<\frac{1}{3} \mu_{0}\right)$ leads flows to evolve to the coexisting steady AC flow rather than the well-behaved smooth subcritical flow.

The long-wave formulation (2.9) is valuable for unsteady flows as it can be straightforwardly numerically integrated with high accuracy to large times, contrasting with 
the $\mathrm{CD}$ integrations of $\mathrm{HJH}$ which become prohibitively computationally intensive as the disturbance to the interface lengthens. CD simulations can, however, follow flows after the interface overturns, as it will in flows forced by sufficiently large perturbations ( $\epsilon$ comparable to $Y_{0}$ or $\left.1-Y_{0}\right)$ of sufficiently small scale $(\mu \sim 1)$. The three overturning sites are within a downstream-propagating bore, at the upstream wave of what would otherwise be a steady lee wavetrain, and during the formation of some $\mathrm{AC}$ flows. In $\mathrm{CD}$ integrations eddies forming on a downstream-propagating bore are swept away without affecting the flow near the topography whereas large eddies formed in a steady lee wavetrain remain near the topography and alter the flow field. In both these cases the long-wave problem (2.9) can be integrated without difficulty provided the resolution of the computation is sufficiently high. Filtering out the short-scale components regularizes the evolution of the interface. This seems to give an accurate description of the long-time behaviour when the CD eddies are swept downstream but may be inaccurate when the eddies remain near the topography. The overturning during the formation of some $\mathrm{AC}$ flows is more troublesome both here and in the CD simulations and appears for large $\epsilon$ even when dispersion is small. As noted in $\S 5.2$, HJH11 shows the transition associated with an initially CC flow steepening as it move slowly downstream to eventually overturn close to the wall $y=0$. Integration of the long-wave problem for these parameters (figure 13) fails when the steepening interface attempts, as it moves downstream, to pass through the wall $y=0$. Here filtering out the short scales precludes following the evolution further. To carry the CD integrations to very large times HJH found it necessary to replace the downstream wavetrain at some large intermediate time with a smooth transition. The evolution could then be followed to a monotonic steady state closely agreeing with the hydraulic solution (figure 3). Vanishingly small frictional effects could lead however to flows in this parameter regime departing from the $\mathrm{CD}$ integration. In discussing AC flows in two-layer non-rotating flow over topography Lawrence (1993) suggests that friction may cause a transition to evolve to a hydraulic jump upstream of the point at which the steady SL of the AC flow would be expected to form. It is not straightforward to include frictional effects in the CD integrations but they could be added to the long-wave model in the same way as to the forced $\mathrm{KdV}$ equation in Smyth (1988).

The dispersive hydraulic method should prove useful in other flows where hydraulic theory has been applied. It has been previously used by Grimshaw \& Yi (1990) and the authors to study finite-amplitude waves on coastal currents. Indeed, in the present geometry in the limit $Y_{0} \rightarrow 0,1$ and $\epsilon \rightarrow 0$ the appropriate cross-channel length scale becomes the shelf width rather than the channel width and the analysis approaches that of Clarke \& Johnson $(1997 a, b)$.

The UK Natural Environment Research Council supported this work under grant number GR3/09174. We are grateful to the referees whose comments let us improve the presentation of this work.

\section{Appendix. Numerical determination of steady solutions}

Steady solutions of the governing equation (2.9) can be rapidly and accurately evaluated by a Newton iteration. Equation (2.9) can be written in the form

$$
\Psi_{h}\left(Y, Y_{h}\right)+\frac{\mu}{6} \Psi_{1}\left(Y, Y_{h}\right)-\frac{\mu}{3} Y(1-Y) \frac{\partial}{\partial \chi}\left[Y(1-Y) Y_{\chi}\right]=\psi_{0},
$$


where $\Psi_{h}\left(Y, Y_{h}\right)$ and $\Psi_{1}\left(Y, Y_{h}\right)$ are low-order polynomials in $Y$ and the derivatives of $Y_{h}$ are known analytically. Take $N$ evenly spaced points $\chi_{i}(i=1, \ldots, N)$ and let $y_{i}$ and $F_{i}$ be the corresponding values of $Y(\chi)$ and the residuals of equation (A 1) at these points (with the derivatives of $Y$ in (A 1) approximated by central differences). Then the discrete form of (A 1) is

$$
\boldsymbol{F}(\boldsymbol{y})=0,
$$

and the corresponding Newton iteration

$$
\boldsymbol{y}^{(n+1)}=\boldsymbol{y}^{(n)}+\hat{\boldsymbol{y}}^{(n)},
$$

where

$$
\boldsymbol{T} \hat{\boldsymbol{y}}^{(n)}=-\boldsymbol{F}\left(\boldsymbol{y}^{(n)}\right) .
$$

The non-zero elements of the tridiagonal Jacobian matrix $\boldsymbol{T}$ follow easily by direct differentiation as

$$
\begin{aligned}
T_{i-1, i}= & -\frac{\mu}{3} y_{i}\left(1-y_{i}\right)\left[y_{i}\left(1-y_{i}\right) / h^{2}+\left(1-2 y_{i}\right) y_{i}^{\prime} / h\right], \\
T_{i, i}= & \frac{\partial \Psi_{h}}{\partial Y}\left(y_{i}, Y_{h}\right)+\frac{\mu}{6} \frac{\partial \Psi_{1}}{\partial Y}\left(y_{i}, Y_{h}\right)+\frac{2 \mu}{3}\left[y_{i}\left(1-y_{i}\right)\right]^{2} / h^{2} \\
& -\frac{\mu}{3}\left\{2 y_{i}^{\prime \prime}\left[y_{i}\left(1-y_{i}\right)^{2}-\left(1-y_{i}\right) y_{i}^{2}\right]+y_{i}^{\prime 2}\left(1-6 y_{i}+6 y_{i}^{2}\right)\right\}, \\
T_{i+1, i}= & -\frac{\mu}{3} y_{i}\left(1-y_{i}\right)\left[y_{i}\left(1-y_{i}\right) / h^{2}-\left(1-2 y_{i}\right) y_{i}^{\prime} / h\right],
\end{aligned}
$$

where $y_{i}^{\prime}=\left(y_{i+1}-y_{i-1}\right) / 2 h$ and $y_{i}^{\prime \prime}=\left(y_{i+1}-2 y_{i}+y_{i-1}\right) / h^{2}$. The iteration can be readily adapted to a stretched grid, however the tridiagonal inversion is so rapid that no stretching was found necessary even for the thinnest transition regions. Typical computations with 4000 points required approximately ten iterations and a few seconds of CPU time on a PC to converge to an accuracy of $10^{-10}$.

\section{REFERENCES}

BAINES, P. 1984 A unified description of two-layer flow over topography. J. Fluid Mech. 146, 127-167. BAINES, P. 1995 Topographic Effects in Stratified Flows. Cambridge University Press.

Bidlot, J. \& SteRn, M. 1994 Maintenance of continental boundary-layer shear through countergradient vorticity flux in a barotropic model. J. Fluid Mech. 271, 55-85.

Canuto, C., Hussaini, M., Quarteroni, A. \& ZAng, T. 1988 Spectral Methods in Fluid Dynamics. Springer.

Clarke, S. \& Johnson, E. 1997a Topographically-forced long waves on a sheared coastal current. Part 1. The weakly nonlinear response. J. Fluid Mech. 343, 131-151.

Clarke, S. \& Johnson, E. $1997 b$ Topographically-forced long waves on a sheared coastal current. Part 2. Finite-amplitude waves. J. Fluid Mech. 343, 153-168.

Clarke, S. \& Johnson, E. 1999 Finite-amplitude topographic Rossby waves in a channel. Phys. Fluids 11, 107-120 (referred to herein as CJ).

FornberG, B \& Whitham, G. 1978 A numerical and theoretical study of certain nonlinear wave phenomena. Phil. Trans. R. Soc. Lond. A 289, 373-404.

Grimshaw, R. \& SmYth, N. 1986 Resonant flow of a stratified fluid over topography. J. Fluid Mech. 169, 429-464.

GrimshaW, R \& Yi, Z. 1990 Finite-amplitude long waves on coastal currents. J. Phys. Oceanogr. 20, $3-18$

HaYnes, P., Johnson, E. \& HuRst, R. 1993 A simple model of Rossby-wave hydraulic behaviour. J. Fluid Mech. 253, 359-384 (referred to herein as HJH). 
Hughes, R. 1985 a Multiple criticalities in coastal flows. Dyn. Atmos. Oceans 9, 321-340.

Hughes, R. $1985 b$ On inertial currents over a sloping continental shelf. Dyn. Atmos. Oceans 9, 49-73.

Hughes, R. $1986 a$ On the conjugate behaviour of weak along-shore flows. Tellus 38A, 227-284.

Hughes, R. $1986 b$ On the role of criticality in coastal flows over irregular bottom topography. Dyn. Atmos. Oceans 10, 129-147.

Hughes, R. 1987 The role of higher shelf modes in coastal hydraulics. J. Mar. Res. 45, 33-58.

Hughes, R. 1989 The hydraulics of local separation in a coastal current, with application to the Kuriosho meander. J. Phys. Oceanogr. 19, 1809-1820.

JACKsON, E. 1990 Perspectives of Nonlinear Dynamics, I, chap. 3. Cambridge University Press.

JoHnsON, E. 1990 The low-frequency scattering of Kelvin-waves by stepped topography. J. Fluid Mech. 215, 23-44.

JoHnson, E. 1993 The low-frequency scattering of Kelvin-waves by continuous topography. J. Fluid Mech. 248, 173-201.

Johnson, E. \& Clarke, S. 1998 Inviscid vortical boundary layers. In Numerical Methods for Fluid Dynamics (ed. M. Baines \& K. Morton), vol. 7. Oxford University Press.

LAwrENCE, G. 1993 The hydraulics of steady two-layer flow over a fixed obstacle. J. Fluid Mech. 254, 605-633.

Marchant, T. \& Smyth, N. 1990 The extended Korteweg-de Vries equation and the resonant flow of a fluid over topography. J. Fluid Mech. 221, 263-288.

Melville, W. \& Helfrich, K. 1987 Transcritical flow over topography. J. Fluid Mech. 178, 31-52.

Pratt, L. \& Armi, L. 1987 Hydraulic control of flows with nonuniform potential vorticity. J. Phys. Oceanogr. 19, 81-106.

Pratt, L. \& Lundberg, P. 1991 Hydraulics of rotating strait and still flow. Ann. Rev. Fluid Mech. 23, 81-106.

Smyth, N. 1987 Modulation theory for resonant flow over topography. Proc. R. Soc. Lond. A 409, 79-97.

Sмyth, N. 1988 Dissipative effects on the resonant flow of a stratified fluid over topography. J. Fluid Mech. 192, 287-312.

Stern, M. 1991 Blocking an inviscid shear flow. J. Fluid Mech. 227, 449-472.

Stern, M. 1993 Topographic jetogenesis and transitions in straits and along continents. J. Phys. Oceanogr. 23, 846-854.

Thompson, J. \& Stewart, H. 1986 Nonlinear Dynamics and Chaos, chap. 13. Wiley.

Woods, A. 1993 The topographic control of planetry-scale flow. J. Fluid Mech. 247, 603-621. 